\title{
Trivalent PROTACs enhance protein degradation through cooperativity and avidity
}

\author{
Alessio Ciulli ( $\nabla$ a.ciulli@dundee.ac.uk) \\ University of Dundee https://orcid.org/0000-0002-8654-1670
}

Satomi Imaide

University of Dundee https://orcid.org/0000-0003-4372-4693

Kristin Riching

Promega

Vesna Vetma

University of Dundee

Claire Whitworth

University of Dundee https://orcid.org/0000-0003-3174-7652

\section{Scott Hughes}

University of Dundee

Nicole Trainor

University of Dundee

\section{Sarah Mahan}

Promega

Nancy Murphy

Promega corporation

\section{Kwok-Ho Chan}

University of Dundee https://orcid.org/0000-0002-6693-2682

\section{Andrea Testa}

University of Dundee

Chiara Maniaci

University of Dundee

\section{Marjeta Urh}

Promega

\section{Danette Daniels}

Promega (United States) https://orcid.org/0000-0002-7659-3020

\section{Article}

Keywords: proteolysis targeting chimeras, PROTACs, protein degradation, trivalent PROTAC 
Posted Date: December 9th, 2020

DOl: https://doi.org/10.21203/rs.3.rs-108753/v1

License: (c) (i) This work is licensed under a Creative Commons Attribution 4.0 International License. Read Full License

Version of Record: A version of this preprint was published at Nature Chemical Biology on October 21st, 2021. See the published version at https://doi.org/10.1038/s41589-021-00878-4. 


\section{Abstract}

Bivalent small-molecule degraders, or proteolysis targeting chimeras (PROTACs), work by simultaneously binding a target protein and E3 ubiquitin ligase to produce a ternary complex. To drive target ubiquitination and degradation at low catalytic concentrations, degraders must form appropriately positioned complexes of sufficient stability, aided by intra-complex interactions. We hypothesized these molecular recognition features could be enhanced by increasing binding valency. Here we present trivalent PROTACs as a strategy to boost protein degradation. Our design for a trivalent PROTAC consisted of two BET bromodomain inhibitors and an E3 ligase ligand, each separately tethered via a branched linker. In screening, we identified SIM1, a VHL-based PROTAC, as a highly potent BET degrader, capable of low picomolar degradation for all family members, with preference for BRD2. In functional comparison studies to bivalent PROTACs or inhibitors, SIM1 showed more sustained anti-cancer activity across numerous therapeutically relevant cell lines. Biophysical, biochemical, and cellular mechanistic studies showed SIM1 induces conformational changes upon binding to the BET protein to simultaneously engage with high avidity both its bromodomains in a cis intramolecular fashion. The resulting 1:1:1 complex showed positive cooperativity, high stability and prolonged cellular residence time. We provide proof-of-concept for augmenting the binding valency of proximity-induced modalities as a strategy to leverage both cooperativity and avidity within the ternary complex to advance functional outcomes.

\section{Introduction}

Bispecific molecular agents that artificially induce proximity between two proteins are an emerging paradigm of pharmaceutical intervention into biology and medicine ${ }^{1}$. Targeted degradation compounds, conventionally classified as either PROTACs or molecular glues depending on whether bivalent or monovalent for binding, have shown great promise as a new class of chemical probes to study biology and therapeutics for treatment of disease ${ }^{2-4}$. Degraders form a ternary complex, bringing together the target protein and an E3 ubiquitin ligase component, resulting in ubiquitination and subsequent degradation of the target protein via the proteasome. Molecular glues typically engage monovalently either the E3 ligase or the target, and then adventitiously induce highly cooperative recruitment of the noncognate partner in a ternary complex ${ }^{5-9}$. In contrast, PROTACs are traditionally conceived as bifunctional, i.e. composed of two ligands joined by a linker, and as a result can engage the target or E3 component separately in binary complexes, or both target and E3 component simultaneously to form a ternary complex ${ }^{10-12}$. This design feature allows PROTACs not only a wider target scope than molecular glues but also the ability to standardize design with the use of established E3 ligase binders. Indeed, potent PROTAC degraders have been developed to most commonly recruit either the von Hippel-Lindau (VHL) or cereblon (CRBN) E3 ligase against a wide spectrum of protein targets including nuclear ${ }^{13-16}$, cytoplasmic ${ }^{11,17,18}$, membrane-bound ${ }^{19}$, and multi-pass transmembrane proteins ${ }^{20}$. 
Due to their complex mode of action via the ternary complex, PROTACs have shown unexpected advantages compared to the inhibitors of which they are composed. For example, PROTACs can discriminate amongst highly homologous targets ${ }^{21-24}$, and can exhibit much greater potencies than expected, due to a catalytic mechanism of action, which can compensate for low binary binding affinities or poor cellular permeability, and allow for use of weak, non-functional inhibitors to serve as warhead ligands ${ }^{16,22,25,26}$. Furthermore, PROTACs can impact proteins in complexes even though not directly engaged by the degrader via either bystander ubiquitination or destabilization ${ }^{16,27,28}$. Despite the aforementioned advantages and remarkable successes demonstrated, it can be challenging to design PROTACs efficiently, often requiring extensive chemical optimization ${ }^{29,30}$. Unlike inhibitors, degraders must function beyond simple binary engagement. Instead they must work throughout a cascade of events, not only inducing proximity between two proteins which do not natively interact, but also yielding a productive ternary complex which structurally positions the target protein for efficient ubiquitination by the E3 ligase ${ }^{31-33}$. Recent X-ray crystallographic structures and allied biophysical studies of PROTAC ternary complexes have elegantly demonstrated that some PROTAC-mediated ternary complexes are, like molecular glues, capable of cooperative binding, most notably shown for VHL-MZ1-BRD4-BD2 ternary complex ${ }^{21}$. This and subsequent studies have shown how in order to drive productive target ubiquitination and profound target degradation at catalytic low concentrations, degraders need to form complexes of sufficient stability and residence time, which can be enhanced by cooperativity and favourable intra-complex interactions ${ }^{16,21,22,31,33}$. Such optimal "glueing" of molecular recognition features can be challenging to realise with conventional PROTAC degraders that are by definition monovalent at the target of interest. This limits their ability to leverage favourable protein-protein and other stabilizing inter- and intra-molecular interactions within the ternary complex. Indeed, noncooperative or even negatively cooperative PROTAC ternary complexes are often observed, which albeit permissive to downstream protein ubiquitination and degradation, can lead to unfavourable pharmacological properties of degraders such as pronounced hook effect at higher concentration, and can result in slow and incomplete target degradation 17,34 . We thus hypothesized that the molecular recognition process could be aided by multivalency, a phenomenon ubiquitous in biology and of attractive appeal in chemistry ${ }^{35,36}$.

Here, we present the design, synthesis and biological and mechanistic characterization of trivalent PROTACs as a strategy to boost targeted protein degradation. We elected the Bromo and Extra Terminal (BET) domain family member proteins BRD2, BRD3 and BRD4 as ideal model systems for this study because numerous well-characterized inhibitors, both monovalent and bivalent binders of their two bromodomains (BD1 and BD2), have been developed, fuelled by the therapeutic relevance of these targets in disease including cancer ${ }^{37-39}$. Several BET family PROTACs, including the afore-mentioned MZ1, have been developed from parent monovalent BET inhibitors ${ }^{10,40,41}$. Altogether, these compounds provided suitable benchmark reference inhibitors and degraders for our study, where we set out to synergize the effects of a bivalent target ligand with E3 ligase recruitment to produce a trivalent PROTAC with enhanced target degradation. These novel PROTACs were further characterized in a series of 
biochemical, biophysical and cellular assays to understand potency, kinetics and specificity of degradation as compared to bivalent PROTAC molecules, as well as for functional outcomes and underlying mechanism of action.

\section{Results}

\section{Structure-guided design and synthesis of trivalent PROTACs}

To design trivalent PROTACs we leveraged our knowledge in both structural biology and pharmacology of parent bivalent compounds. Insight into a strategy for chemical design came first from analysis of the crystal structure of $M Z 11^{10}$, a BET PROTAC composed of VHL ligand VH032 conjugated to pan-BET BD inhibitor (+)-JQ1, bound in a ternary complex with VHL and the second bromodomain of BRD4, BRD4 ${ }^{\mathrm{BD} 2}$

${ }^{21}$. The structure identified the central portion of the PEG3 linker as solvent exposed, suggesting it as a branching point to link to a second BET ligand (Fig. 1a). Similarly, inspection of the co-crystal structure of the bivalent BET inhibitor MT1, a homodimer of (+)-JQ1 with a PEG7 linker, bound to two monomers of $B R D 4$ BD2 42 , revealed a site within the central region of the linker for potential chemical branching towards an E3 ligase ligand (Fig. 1b). In contrast, the bivalent inhibitor Bi-BET is fully buried at the interface of the two bromodomains in its co-crystal structure ${ }^{43}$, suggesting derivatization could impair its binding mode (Extended Data Fig. 1a). We therefore elected MZ1 and MT1 as the progenitor bifunctional molecules in our design strategy.

We next imagined that a trifunctional PROTAC could be assembled around a 'core scaffold' connected to each of its three ligands via variable linkers (Fig. 1c). We reasoned that 1,1,1-tris(hydroxymethyl)ethane, also known as trimethylolethane (TME), could provide fit-for-purpose scaffold because it features three primary alcohol groups in a neopentyl core structure, thus acting as branched, close bioisosteric replacements of PEG units. We envisaged that achirality of the central quaternary carbon of the TME core could be readily achieved by keeping the chemical linkers to each BET ligand moiety identical. To allow flexibility in exploring the relative constraints between the different monomeric ligands, while at the same time keeping the overall chemical structure as close as possible to those of MZ1 and MT1, we designed three branched PROTACs (SIM1-SIM3) bearing PEG3 or PEG4 at each linker to the BET ligand $(n=3,4)$, and PEGO or PEG1 towards VH032 ( $m=0,1)$ (Fig. 1c). We also designed analogous compounds composed of the CRBN targeting ligand pomalidomide in place of VH032 (SIM4-SIM6) (Fig. 1c).

To synthesize VHL-based trivalent PROTACs (general structure 12) we started from the acetonide 1, synthesized from TME, as key precursor to the trivalent linker core (Scheme 1). Allylation of 1 and subsequent deprotection gave diol $\mathbf{2}$, which was alkylated with the mesylate linker $\mathbf{3}$ to give the allyl ether 4 as the precursor for PROTACs bearing $m=0$ to the E3 ligase ligand. To introduce the additional PEG1 unit $(m=1)$, alcohol 1 was reacted with iodide 5 in the presence of sodium hydride. Deprotection of the acetonide group of $\mathbf{6}$ and subsequent alkylation of the revealed primary alcohols with linker $\mathbf{3}$ yielded the di-azide 7. Deprotection of the p-methoxybenzyl (PMB) group of 7 with 2,3-dichloro-5,6-dicyano-1,4- 
benzoquinone (DDQ) and subsequent allylation afforded allyl ether 8. Oxidative cleavage of the double bond of the allyl ethers $\mathbf{4}$ or $\mathbf{8}$ and subsequent Pinnick oxidation of the resulting aldehyde $\mathbf{9}$ gave the carboxylic acid 10, which was coupled with VH032-amine ${ }^{27,44}$. Reduction of the two azide groups in $\mathbf{1 1}$ to amine and further amide coupling with (+)-JQ1 carboxylic acid, completed the preparation of VHLbased trivalent PROTACs. CRBN-based trivalent PROTAC were similarly achieved by coupling an aminecontaining analogue of pomalidomide ${ }^{29}$, in place of VH032-amine (Supplementary Note).

\section{SIM1 is a potent VHL-based trivalent PROTAC with preference for BRD2 degradation}

To evaluate the ability of trivalent compounds to induce intracellular degradation of BET proteins, we first treated human HEK293 cells for $4 \mathrm{~h}$ at $1 \mu \mathrm{M}$ concentration and assessed levels of BRD2, BRD3 and BRD4 by western blot. Profound degradation across BET proteins was observed with VHL-based SIM1-SIM3, whilst minimal to no-degradation was observed with CRBN-based SIM4-SIM6 (Fig. 1d). To confirm the greater activity of VHL-based compounds compared to CRBN-based PROTACs we next used live cell continual luminescent monitoring of CRISPR/Cas9 endogenously tagged HiBiT-BRD4 in HEK293 cells over $24 \mathrm{~h}^{31}$. Faster initial rates of BRD4 degradation accompanied by greater maximal degradation levels were seen with SIM1-SIM3 as compared to SIM4-SIM6 (Fig. 1e). Dose response kinetic analysis of HiBiTBRD 4 with SIM1-SIM3 showed rapid and complete degradation from $4 \mathrm{nM}-1 \mu \mathrm{M}$ with no observable hook effect for any of the compounds (Extended Data Fig. 1b). To confirm greater potency of VHL-based compounds, we evaluated growth inhibition profiles of BET-sensitive cancer cell lines MV4;11 (Fig. 1f), A549 and HL-60 (Extended Data Fig. 1c) with compound treatments. SIM1-SIM3 consistently showed more potent activity relative to SIM4-SIM6, as well as bifunctional molecules MZ1 and parent MT1 (Fig. $1 \mathrm{f}$ and Extended Data Fig. 1c). Together, the initial screens support greater activity of structureguided designed VHL degraders over their homologous CRBN molecules.

Encouraged by these initial results we next sought to characterize in more depth the degradation activity of the VHL-based degraders across the BET protein family. Concentration-dependent profiling at $4 \mathrm{~h}$ treatments using immunoblots evidenced much lower $\mathrm{DC}_{50}$ values of $0.7-9.5 \mathrm{nM}$ for SIM1-SIM3 compared to MZ1 ( DC $_{50}$ values of 25-920 nM) across all the BET proteins, with SIM1 emerging as the most potent of the three degraders (Fig. 2a and Extended Data Fig. 1d). We also observed a preference for degradation of BRD2 over BRD3 and BRD4 with all compounds, compared to the marked preferential BRD4 degradation of MZ1 (Extended Data Fig. 1d) 10,21,25. Quantitative live-cell kinetic degradation dose response curves were performed with CRISPR HiBiT-BET HEK293 cells with SIM1-SIM3 titrations up to $10 \mathrm{nM}$ concentration (Fig. 2b and Extended Data Fig. 2a,b). These analyses further qualified SIM1 as the most potent degrader across all BET family members (Dmax ${ }_{50} 60-400$ pM) (Fig. 2b,c and Extended Data Fig. 2a-c), with BRD2 showing the fastest degradation rate and lowest Dmax $_{50}$ values across all SIM1SIM3 compounds (Fig. 2c and Extended Data Fig. 2c). 
To assess the cellular selectivity of SIM1 for BET proteins, multiplexed tandem mass tag (TMT) labeling mass spectrometry proteomic experiments were performed to monitor protein levels in a quantitative and unbiased fashion. To furnish a suitable non-degrading negative control isomer in these experiments, we synthesized compound cis-SIM1 bearing inverted stereochemistry at the hydroxyproline centre of the VHL ligand resulting in loss of binding to VHL (Supplementary Note) ${ }^{10,12}$. Acute myeloid leukemia (AML) MV4;11 cells were treated in triplicate with DMSO, $10 \mathrm{nM} \mathrm{SIM1,} \mathrm{or} 10 \mathrm{nM}$ cis-SIM1 for $4 \mathrm{~h}$. Among the 5,232 proteins quantified, BRD2 was found as most significantly degraded by SIM1, followed by BRD3 and BRD4 (Fig. 2d and Extended Data Fig. 2d). No significant changes in BET protein abundance were observed in cells treated with cis-SIM1. We also observed SIM1 induced decrease in protein levels for Myc, a known downstream effect of BET-induced degradation, and HMOX1 (heme oxygenase 1) suggesting early initiation of apoptosis ${ }^{45}$.

As enhanced rate and/or levels of degradation have been found to correlate with enhanced ubiquitination, cellular studies were performed to monitor the kinetics of ubiquitination of BET family members using bioluminescence resonance energy transfer (NanoBRET) assays consisting of the HiBiTBET CRISPR cell lines expressing fluorescently labelled HaloTag-Ubiquitin ${ }^{31}$. Shown in Fig. 2e, the kinetic increase in cellular ubiquitination was more robust for BRD2 as compared to BRD3 and BRD4 after a $10 \mathrm{nM}$ SIM1 treatment. These same trends were observed at $100 \mathrm{nM}$ SIM1 concentration and comparison to MZ1, revealed SIM1 led to higher levels of ubiquitination of all BET family members, with the greatest difference observed for BRD2 (Extended Data Fig. 2e). Together, these cellular degradation data highlighted SIM1 as the most potent BET degrader amongst the studied trivalent PROTACs, with preferential degradation of BRD2.

\section{SIM1 is functionally more potent than bivalent BET PROTACs and parent bifunctional inhibitors}

To determine whether increase in target binding valency of SIM1 improved both degradation and efficacy as compared to other VHL-based bivalent PROTACs, kinetic degradation dose response profiles for BRD2, BRD3, and BRD4 were first determined for the bivalent BET PROTAC ARV-771 ${ }^{40}$ (Extended Data Fig. 3a). Calculated from these and the previously published MZ1 analysis ${ }^{31}$, degradation rates and Dmax $_{50}$ values as compared to SIM1 were plotted for BRD2 (Fig. 3a), as well as BRD3 and BRD4 (Extended Data Fig. 3b). These revealed SIM1 treatment vastly improved rates of BET family degradation as well as Dmax $_{50}$ values, ranging from increases in degradation potency of 80-300X for BRD2 (Fig. 3a), 2-35X for BRD3, and 10-20X for BRD4 (Extended Data Fig. 3b). To understand if the improvement in degradation of the trivalent PROTAC relative to bivalent BET PROTACs translated to enhanced functional outcomes, we next moved to study of SIM1 compound response in a BET-sensitive cell line, the prostate cancer line 22Rv1. Treatment of 22Rv1 cells with varying concentrations of compounds at the early time point of $4 \mathrm{~h}$ confirmed the enhancement in BET degradation potency and cMyc level suppression activity of SIM1 compared to related bifunctional BET degraders MZ1 and ARV-771, as well as non-degrading controls 
MT1 and cis-SIM1 (Fig. 3b and Extended Data Fig. 3c). The expected mechanistic dependency of SIM1induced degradation on functional VHL E3 ligase and proteasomal activity was confirmed with cotreatment with VHL inhibitor VH298 ${ }^{44}$ and proteasome inhibitor MG132 (Extended Data Fig. 3d).

BET inhibitors and degraders result in down-regulation of numerous targets, including cMyc, which showed loss after $4 \mathrm{~h}$ treatment with $10 \mathrm{nM}$ SIM1 in unbiased proteomics profiling in the BET-relevant AML line of MV4;11 (Fig. 2d). To quantitate the time-dependent loss of cMyc in this cancer cell line, cMyc was endogenously tagged with $\mathrm{HiBiT}$ and protein levels were monitored in cell lysates at varying times with different concentrations of SIM1, cis-SIM1, and MT1 (Fig. 3c, left and Extended Data Fig. 4a). Rapid and complete loss of cMyc was observed with $1 \mathrm{nM}$ SIM1 concentration (Fig. 3c, left) whereas similar levels of cMyc loss with the parent MT1 or cis-SIM1 were not achieved until 50-100 nM treatments (Extended Data Fig. 4a). Correlative cell viability assays were performed to understand how each compound impacted cell death, and these revealed that only treatment with SIM1 at $1 \mathrm{nM}$ (and not cisSIM1 or MT1) resulted in measurable cellular death after $6 \mathrm{~h}$ (Fig. 3c, right). Similar trends were observed at higher compounds concentrations where SIM1 was found to be significantly more cytotoxic than control compounds at any concentration after 6 h (Extended Data Fig. 4b)

Next, we assessed the functional effects of SIM1 on the viability of 22Rv1 cancer cells. Substantial cell death was observed after $24 \mathrm{~h}$ treatment with $10 \mathrm{nM}$ SIM1, as shown by PARP cleavage assays (Fig. 3d and Extended Data Fig. 5a). In contrast, MT1 or MZ1 did not cause observable PARP cleavage even up to $48 \mathrm{~h}$ treatment at the same concentration as SIM1 (Extended Data Fig. 5b-c). Similar levels of PARP cleavage were observed with MT1 and MZ1 only at $1 \mu \mathrm{M}$ concentration (Extended Data Fig. 5b-c). Cells died of apoptosis as indicated by prevented cleavage of PARP upon co-treatment with QVD-OPh, a pancaspase inhibitor, and not of necroptosis, as shown by co-treatment with Necrostatin-1 (Fig. 3d and Extended Data Fig. 5a). Caspase-Glo assays confirmed the significantly more potent activity of SIM1 $\left(E_{50} 2 \mathrm{nM}\right)$ compared to bifunctional degraders MZ1 and ARV-771 ( $\mathrm{EC}_{50} 150$ and $90 \mathrm{nM}$, respectively), with much greater maximal signal than non-degraders cis-SIM1 and MT1 (Fig. 3e). Caspase-Glo activity of SIM1, MZ1, and ARV-771 was blocked by co-treatment with the VH298 and QVD-OPh, whereas only QVD-OPh and not VH298 co-treatment blocked activity of cis-SIM1 and MT1, consistent with their different mode of action (Extended Data Fig. 5d). Finally, the superior activity of SIM1 was evidenced in a colony-formation assay in 22Rv1 cells. After an initial treatment with test compounds at $10 \mathrm{nM}$ concentration, followed by 20 days of growth, only the SIM1-treated cells showed significant cytotoxicity as compared to vehicle control (Fig. 3f). Together, the biological data supports more potent degradation and more substantial downstream functional activity of the trivalent degrader SIM1 compared to benchmark parent bivalent degraders or inhibitors.

\section{SIM1 engages BD1 and BD2 intramolecularly and forms a 1:1:1 ternary complex with VHL and BRD4}


The remarkable cellular activity of SIM1 prompted us to interrogate the molecular recognition of trivalent PROTAC complexes with the VHL E3 ligase and BRD4 as representative BET protein. Previous work had established that bivalent BET inhibitors simultaneously engage BD1 and BD2 bromodomains within a BET protein ${ }^{42,43}$. We thus hypothesized that trivalent BET PROTAC SIM1 would also display a cis intramolecular engagement of the BET protein. To address this, we first employed biophysical binding assays with recombinant proteins. We performed size-exclusion chromatography (SEC) ${ }^{27}$ experiments using tandem BD1-BD2 constructs from BRD4 that were either wild-type (WT), so competent to cis intramolecular binding, or contained a point mutation in either BD1 or BD2 at a conserved asparagine residue in the ligand-binding pocket ( $\mathrm{N} 140 \mathrm{~F}$ in $\mathrm{BRD} 4^{\mathrm{BD} 1}$, or $\mathrm{N} 433 \mathrm{~F}$ in $\mathrm{BRD} 4^{\mathrm{BD} 2}$ ), to abrogate binding to one of the two bromodomains thus rendering the mutant tandem unable to engage in a cis bivalent fashion ${ }^{43}$. SIM1 was found to shift the SEC profile of BRD4 WT BD1-BD2 tandem construct to a higher elution volume, compared to free or MZ1-bound BRD4, consistent with the formation of a more compact intramolecular 1:1 complex with SIM1, as observed with MT1 (Fig. 4a). In contrast, when either N140F or $\mathrm{N} 433 \mathrm{~F}$ mutant tandems were used, we observed a significant shift to lower elution volumes with both SIM1 and MT1, compared to free or MZ1-bound BRD4, consistent with the formation of higher-molecular weight 2:1 species in solution (Fig. 4a and Extended Data Fig. 6a). Having established that SIM1 engages BD1 and BD2 in a cis fashion, using its bivalent BET ligand portion, we next asked whether SIM1 could then form the expected 1:1:1 complex between the BD1-BD2 tandem domain and the E3 ligase VHL, by utilizing the remaining binding valency from the VHL ligand portion. Indeed, a sample containing 1:1:1 equivalents of SIM1, BD1-BD2, and VHL-ElonginB-ElonginC complex (VCB) ran at lower elution volumes compared to either of the two peaks observed from a sample containing the same equivalent ratio of cisSIM1, BD1-BD2 tandem and VCB, where only the 1:1 cis-SIM1:BD1-BD2 complex and unbound VCB can be formed (Fig. 4a).

Previous biophysical and cellular studies with bivalent BET inhibitors, showed that the intramolecular binding of BD1 and BD2 resulted in a structural conformational change of BRD4 ${ }^{43}$. To determine if SIM1 cellular binding to BRD4 could also induce a structural change, we utilized a NanoBRET biosensor containing the same BD1-BD2 tandem domains of WT BRD4 or mutant N433F, flanked respectively by a NanoLuc donor and HaloTag acceptor (Fig. 4b). With the BD1-BD2 tandem WT biosensor, compounds SIM1, cis-SIM1 and MT1 all showed a change in BRD4 conformation, manifested by an increase in NanoBRET signal which reached and maintained a plateau, as is expected for an intramolecular engagement (Fig. 4b). As a control, the BD1-BD2 N433F mutant sensor showed no response indicating that simultaneous binding of BD1 and BD2 is required for the conformation change (Fig. 4b).

Interestingly cis-SIM1 and SIM1 showed higher $\mathrm{EC}_{50}$ values for BRD4 engagement than MT1 (Fig. 4b). To determine if this is due to a reduced binding affinity of BRD4 and/or reduced permeability given their increase in molecular weight, NanoBRET target engagement assays were performed measuring displacement of a fluorescent BET tracer molecule bound to HiBiT-BRD4 ${ }^{43}$. In permeabilized cells, where permeability is not a factor, we observed binding of SIM1, cis-SIM1, and MT1 to endogenous HiBiT-BRD4 with near-identical binding affinities and $I_{50}$ values (Extended Data Fig. 6 b). However in live cells, SIM1 (and to a lesser extent cis-SIM1) showed reduced binding affinity to BRD4 compared to MT1, suggesting 
the $\mathrm{EC}_{50}$ shift observed in the conformational sensor assay reflects reduced permeability of the trivalent molecule relative to the parent bivalent inhibitor (Extended Data Fig. 6b). Despite this reduced cellular permeability, SIM1 is a highly potent degrader and these biochemical and cellular studies give insights as to its mode of ternary complex formation, wherein SIM1 simultaneously engages BD1 and BD2 within a given BET protein, and recruits VHL to form a 1:1:1 complex.

\section{SIM1 forms cooperative stable ternary complexes with enhanced cellular residence time}

To validate the ternary complex stoichiometry and further characterize their binding thermodynamics, we next used isothermal titration calorimetry (ITC) by performing reverse titrations ${ }^{21}$. First, in titrations of BRD 4 N433F or N140F tandems (competent for BD1 or BD2 binding alone, respectively) into preformed SIM1:VCB complex we observed 2:1 stoichiometry, molar binding enthalpy of $\Delta \mathrm{H}=-11.6$ and $9.1 \mathrm{kcal} / \mathrm{mol}$, and $K_{\mathrm{d}}=1.2$ and $0.12 \mu \mathrm{M}$, respectively. In contrast, titration of BRD4 WT BD1-BD2 under identical conditions displayed binding stoichiometry of 1:1, a large negative binding enthalpy $(\Delta \mathrm{H}=-$ $20 \mathrm{kcal} / \mathrm{mol})$, and avidity of binding $\left(K_{\mathrm{d}}<20 \mathrm{nM}\right)$ (Fig. 4c). Together, the ITC data confirmed the stoichiometries observed in SEC and point to preferential engagement of BRD4-BD2 by VCB:SIM1 prior to intramolecular 1:1 engagement. To study the favourability of engaging both BD1 and BD2 in ternary complex with VHL in the context of cellular, full-length BRD4, we interrogated VHL binding to full-length BRD4 WT, N140F, or N433F mutations using kinetic NanoBRET ternary complex assays ${ }^{31}$. HEK293 cells expressing the various NanoLuc-BRD4 fusions with HaloTag-VHL showed ternary complex with VHL and WT BRD4 was rapidly formed in the presence of SIM1, but not with controls cis-SIM1 or MT1 (Fig. 4d). Ternary complex formation however was markedly reduced with the BRD4 N140F mutation and almost abolished with the N433F mutation (Fig. 4d). These results confirm SIM1 utilizes both BD1 and BD2 for ternary complex formation and suggest preferential binding to BD2 over BD1, consistent with the ITC results and what was found previously with MZ1 ${ }^{21,33}$. Cellular NanoBRET ternary complex formation with SIM1 compared to MZ1 was also assessed with the panel of endogenously tagged HiBiT BET family members and a more robust and sustained ternary complex was observed for BRD2 and BRD4 with VHL induced by SIM1 as compared to MZ1, while that with BRD3 did not appear to be as prolonged or stable (Extended Data Fig. 6c).

We next asked to what extent SIM1 might exhibit cooperativity of ternary complex engagement, over and above its avidity of bivalent BET binding. SIM1 exhibited a positive cooperativity a value of 3.5 for ternary complex formation as shown in competitive AlphaLISA assays (Fig. 4e), measuring binding of SIM1 alone $\left(\mathrm{IC}_{50}=205 \mathrm{nM}\right)$ or SIM1:VCB binary complex $\left(\mathrm{IC}_{50}=58 \mathrm{nM}\right)$ via the displacement of biotin-JQ1 46 from BRD4. As a cross- validation, we evaluated cooperativity in a competitive FP assay measuring binding at the VHL end of the PROTAC molecule via the displacement of a fluorescent HIF-1 a peptide probe ${ }^{44}$. Again, in this experiment SIM1 exhibited positive cooperativity $(a=5.2)$ from enhanced affinity of its competitive displacement as binary SIM1:BD1-BD2 tandem $\left(K_{d}=33 \mathrm{nM}\right)$ compared to SIM1 alone 
$\left(K_{d}=186 \mathrm{nM}\right)($ Fig. 4f). We next assessed kinetics of dissociation of ternary complexes of SIM1, first in an SPR binding assay ${ }^{33}$. We immobilized biotinylated VCB onto the surface chip and injected seriallydiluted solution of SIM1 pre-incubated in excess of BD1-BD2 protein, in a single-cycle kinetic format experiment (Fig. 4g) ${ }^{33}$. SIM1:BD1-BD2 bound to VCB in 1:1 stoichiometry, as evidenced by the experimental \% Rmax value for the expected $1: 1$ binding model $(64.7 \pm 2.3 \%)$ being comparable to and not exceeding that observed from a reference titration of SIM1 alone (77\%) (Extended Data Table 1). SIM1 formed a high-affinity, stable and long-lived 1:1:1 complex with wild-type BD1-BD2 and VCB $\left(t_{1 / 2}=\right.$ $119 \pm 21 \mathrm{~s} ; K_{\mathrm{d}}=53 \pm 4 \mathrm{nM}$ ) (Fig. $4 \mathrm{~g}$ and Extended Data Table 1). The positive cooperativity and stability of SIM1 ternary complexes with recombinant proteins prompted us next to interrogate its ternary complex residence time in live cells, relative to parent monovalent and bivalent molecules. To monitor residence time, the HiBiT-BRD4 CRISPR cells were first incubated with saturating concentrations of PROTAC or inhibitor compounds, followed by the competitive BET fluorescent tracer. The NanoBRET signal produced from this displacement can be monitored kinetically in live cells, the rate and intensity of which directly correlates to the residence time of the initial compound-bound complex. In agreement with previous studies, JQ1 had a short residence time matching the tracer alone which is derived from a similar monovalent BET inhibitor ${ }^{43}$ (Fig. 4h), while MT1 showed longer residence time, as expected for a bivalent inhibitor. Interestingly MZ1 showed a residence time between the monovalent and bivalent BET inhibitors, indicating even with monovalent BET target engagement, improvements in residence time are possible with a PROTAC (Fig. 4h), which we ascribe to its cooperative binding ${ }^{21}$. Striking differences were observed between cis-SIM1 and SIM1, with SIM1 showing the longest residence time on BRD4 while cisSIM1 showed identical displacement kinetics as MT1 (Fig. 4h). The different behaviours between cisSIM1 and SIM1, which both exhibit avidity from bivalent target engagement, indicate that recruitment of VHL by SIM1 significantly increases the residence time on BRD4, due to the formation of a highly stable and cooperative ternary complex inside the cell. Together, the results from these biophysical binding assays and cellular studies in vitro demonstrate SIM1 forms cooperative, stable and long-lived 1:1:1 ternary complex with VHL and BET proteins. Furthermore, our findings suggest this is facilitated by intramolecular binding that results in both a structural change as well as prolonged compound interaction with the target, which is even further enhanced by the cooperative engagement of VHL in the ternary complex.

\section{Discussion}

The study presented here finds inspiration from an often-used strategy in chemical biology and drug discovery for improvement of compound efficacy, i.e. increasing target binding valency, and has leveraged this for improved PROTAC-mediated targeted protein degradation. Our work qualifies the novel trivalent PROTAC SIM1 as a profoundly potent and fast degrader of BET proteins. Our biological and mechanistic investigation with SIM1 provides proof-of-concept for augmenting the valency of degraders as a viable strategy to boost their mode of action by positively impacting the ternary complex. To understand the mechanism of action of SIM1, a series of biophysical, biochemical, and cellular studies were performed. Most critically these showed a 1:1:1 VHL:SIM1:BRD4 complex stoichiometry and SIM1 
bound intramolecularly in a cis-fashion to both BD1 and BD2 of BRD4, inducing a conformational change (Fig. 5a). Further investigation with BRD4 BD1 or BD2 mutants suggested there is preferred BD binding of SIM1 into BRD4, with BD2 being the first step (Fig. 5a), consistent with the BD2-specificity of the parent PROTAC MZ1 21,33. Based upon the data presented here and degradation patterns of all BET family members, we thus propose all BET family engage with SIM1 in this fashion. Interestingly BRD2 was found in a series of orthogonal assays to show the highest level of degradation and correspondingly the most robust level of ubiquitination of the family members with all VHL-based SIM1-SIM3, which to our knowledge is unprecedented preference for BRD2 from this class of BET PROTAC degraders. It is not known why there is this preference, and we speculate this could reflect greater kinetic favourability of ternary complex formation of BRD2 with VHL, or the possibility that the induced structural change better positions BRD2 in a more favourable state for more productive ubiquitination, as compared to BRD3 or BRD4.

For successful protein degradation to occur, the induced ternary complex between the target protein and the E3 ligase not only must form, but the target within the complex must also be well positioned for efficient ubiquitination. To achieve this structural favorability, numerous linkers are tested in the development of PROTACs to discover compounds which optimally recruit the target in a position and geometry productive for E2/E3 catalyzed neo-substrate ubiquitination 21,34,47. An additional factor which can be crucial for success, perhaps even overcoming non-optimal structural positioning, is thermodynamic and kinetic favorability of ternary complex formation. In these cases, PROTACs facilitate positive neo-interactions between the E3 ligase and target, similar to the mechanism of monovalent molecular glues, resulting in stable, cooperative, and long-lived ternary complexes which drive catalytic ubiquitination $21,31,33,48$. For compounds which do not have this latter ternary complex favorability, their window of degradation efficacy will be limited by the hook effect as the binary complexes will have higher preference of formation than the ternary complex (Fig. 5b) ${ }^{17,49}$. On the other hand, PROTACs which do facilitate cooperative ternary complexes will have a broader window of degradation activity as the complex equilibria is now shifted towards the ternary complex as compared to the non-productive binary interactions of compounds with either the target or E3 ligase (Fig. 5b). The downstream ubiquitination and degradation outcomes will be ternary complex dependent and therefore could potentially be optimized through rational chemical design ${ }^{16,21}$. In our studies with the trivalent PROTAC, we provide evidence that optimization of structural, energetic and kinetic ternary complex favorability parameters occurred from combined cooperativity and avidity in the process (Fig. 5b). Our data suggests that it is this combination which boosted most significantly degradation activity, as the trivalent CRBNbased PROTACs, which also would have avidity, were much less active. The highly stabilized ternary complex is most notably observed with the prolonged cellular residence time of SIM1 binding to BRD4, particularly when VHL can engage to form the ternary complex. An improvement in all the parameters with the trivalent PROTAC resulted in a vast expansion of the degradation window, from rapid rates of BET family loss at very low concentration to maximal degradation with no hook effect observed at higher concentration 10,000-fold above the $\operatorname{Dmax}_{50}$. Some of these characteristics have been observed previously with bivalent BET family PROTACs, such as cooperative complexes with degrader MZ1 10,21 
and potent degradation with dBET- $6{ }^{41}$ and ARV- $771^{40}$, but the combination of these to achieve maximal avidity in the ternary complex to drive profound degradation had not yet been shown.

The transition from heterobifunctional degraders to trivalent degraders might not immediately seem like an obvious approach for improvement of degradation outcomes, particularly given the chemical synthesis challenges and presupposition that increasing molecular weight of degraders would be accompanied by either decreased cellular permeability or a completely impermeable compound. However, with the SIM compounds shown and characterized here, we have demonstrated that this is not the case. While indeed the trivalent SIM1 and cis-SIM1 compounds have reduced permeability compared to their parent bivalent inhibitor, they are still readily cell-permeable, which was sufficient to allow them to outperform parent bivalent BET inhibitors and PROTACs in relevant cellular disease assays used for assessment of BET compound potential for therapeutic use. As such, the enhanced activity and increased sites of binding of trivalent PROTACs might allow to alleviate or circumvent cancer resistance mechanisms that are beginning to emerge with conventional monovalent and bivalent degraders ${ }^{50-52}$.

While the chemical design and synthesis of a trivalent degrader is more involved than for bivalent PROTACs, the increased effort showed significant benefits and allowed to overcome these perceived challenges, affording a much-improved degrader. To achieve this, outlined is a new linker design strategy for generation of a branched trifunctional scaffold to which both target and E3 binders could be conjugated. This chemical approach of augmenting valency provides highly modular design and opens to numerous future applications (Fig. 5c). We envision the potential of multivalent PROTAC development, beyond a "rule of 2", as a new strategy of utility and development to enhance not only for targeted protein degradation mechanisms, but as a means to expand multi-specific compounds. Though we have shown here increased valency to address two repeat domains within a target, trifunctional compounds could be directed towards either two distinct domains on the same protein or even two distinct proteins (Fig. 5c). One immediate example where trifunctional compounds could be applied towards distinct domains within a target would be epigenetic proteins, which frequently contain tandem histone modifying or binding domains that are specifically ligandable ${ }^{53}$. Applications extending to other targets are expected to be of immediate reach where bivalent or multivalent ligands exist, can be identified via hit finding approaches, or can be rationally designed. To broaden scope, multivalent avidity may allow leveraging weak intramolecular binding at the ternary complex interface, as a strategy to selectively stabilise and bias specific conformations of a target protein more amenable to ubiquitination. Multivalent avidity may also allow to best exploit weak binding ligands in their own right, for targeting shallow surfaces or small pockets, for which weak binding fragments can be readily found but can be challenging to optimize as monovalent agents ${ }^{54,55}$.

Initial work with multispecific drug modalities has been transformative for drug discovery and greatly expanded the therapeutic protein landscape ${ }^{1}$. In addition to these compounds being multifunctional, improved pharmacodynamics is likely given avidity and/or neo-interactions driven by the trivalent compounds. This trifunctional compound concept is not limited to E3 ligase recruitment, and could be 
applied to any three protein targets if binding ligands for each are known and structural or geometry constraints allow. An example of this could be two subunits within a multi-protein complex recruited either simultaneously or independently to a biological effector protein such as an E3 ligase, kinase, or phosphatase. A multivalent design concept could find utility beyond PROTACs for emerging approaches for small-molecule induced proximity ${ }^{56-58}$. We thus anticipate broad applicability of the approach to boost mechanisms of action and performance of a wide range of multispecific agents and modalities for chemical biology and pharmaceutical development.

\section{Methods}

Compounds. Synthesis of new compounds and their intermediates is described in the Supplementary Note. MZ1 ${ }^{10}, M T 1^{42}$ and ARV- $771^{40}$ were synthesized following previously described procedures, unless stated otherwise.

Cell lines and culture. HEK293, 22Rv1 and MV4;11 cells (ATCC) were grown in DMEM and RPMI (Invitrogen) respectively and supplemented with $10 \% \mathrm{v} / \mathrm{v}$ fetal bovine serum (FBS) (South American origin, Life Science Production) at $37^{\circ} \mathrm{C}$ and $5 \% \mathrm{CO}_{2}$ in a humidified atmosphere. CRISPR HiBiT-BRD2, HiBiT-BRD3, and HiBiT-BRD4 HEK293 cells stably expressing LgBiT were grown in DMEM with $10 \% \mathrm{v} / \mathrm{v}$ FBS and CRISPR cMyc-HiBiT MV4;11 cells were grown in RPMI with 10\% v/v FBS. All cells were split 1-2 times per week when $90 \%$ confluent and were not used beyond passage 30 . Cells were routinely checked for mycoplasma contamination using Mycoalert detection kit (Lonza).

Vector constructs. Wild-type and mutant versions of human proteins BRD2 (P25440), BRD3 (Q15059) and BRD4 (060885) VHL (UniProt accession number: P40337), ElonginC (Q15369), ElonginB (Q15370), and Ubiquitin UBB (UniProt POCG47) were used for all protein expression.

pET-His-SUMO TEV BRD4 tandem was produced by cloning truncated BRD4 containing the two bromodomains (residue 1-463) into parent pET His6 Sumo TEV LIC cloning vector (1S) using ligase independent cloning. pET His6 Sumo TEV LIC cloning vector (1S) was a gift from Scott Gradia (Addgene plasmid \# 29659). Quikchange mutagenesis was performed on pET-His-SUMO BRD4 tandem with mutagenic primer following standard procedures, to generate mutant with the conserved Asparagine sitting in the Acetyl-lysine binding pocket substituted with Phenylalanine, i.e. BRD4 N140F and BRD4 N433F. For cellular NanoBRET experiments, the following vectors were generated: N-terminal NanoLuc fusions of full-length human BRD4 wild-type (WT), N140F, or N433F, HaloTag-VHL (Promega), HaloTagUBB (Promega), and the biosensor BRD4 NanoLuc-BD1-BD2-HaloTag containing the tandem BD1-BD2 domain (AA 44-460) with a wild-type sequence or N433F mutation.

Degradation assays. MV4;11 cells were seeded at $1 \times 10^{6}$ cells $/ \mathrm{mL}$ of $10 \mathrm{~cm}$ dishes for $12-24 \mathrm{~h}$ before treatment. 22Rv1 and HEK293 cells were seeded at 2.5-6 x 105 cells/well of 6 well plates for 12-24h before treatment. Cells were treated with test compounds with and without inhibitors as indicated or an equivalent volume of DMSO and lysed at the stated time point. For lysis, cells were washed twice in ice 
cold PBS (Invitrogen) then lysed in 250 $\mathrm{L} /$ plate for MV4;11 cells or $80 \mu \mathrm{L} /$ well for 22Rv1 and HEK293 cells of ice cold lysis buffer containing $50 \mathrm{mM}$ Tris hydrochloride $\mathrm{pH} 7.4,150 \mathrm{mM}$ sodium chloride, $1 \mathrm{mM}$ EDTA pH 7.4, 1 \% v/v Triton X-100, $1 \times$ Halt ${ }^{\text {TM }}$ Protease Inhibitor Cocktail (ThermoFisher). Lysates were sonicated, cleared by centrifugation at $4^{\circ} \mathrm{C}$, at $15800 \times \mathrm{g}$ for 10 mins and the supernatants stored at $-80^{\circ} \mathrm{C}$. Protein concentration was determined by BCA assay (Pierce) and the absorbance at $562 \mathrm{~nm}$ measured by spectrophotometry (NanoDrop ND1000). Samples were run on SDS-PAGE using NuPAGE Novex $4-12 \%$ Bis-Tris gels (Invitrogen) with $40 \mu \mathrm{g}$ total protein/well, transferred to $0.2 \mu \mathrm{m}$ pore nitrocellulose membrane (Amersham) by wet transfer and blocked with 3\% w/v BSA (Sigma) in $0.1 \%$ TBST. Blots were incubated in anti-BRD2 (1:2000, abcam \#ab139690), anti-BRD3 (1:500, abcam \#ab50818), anti-BRD4 (1:1000, abcam \#ab128874), anti-c-myc (1:1000, abcam \#32072), anti-PARP (1:1000, CST \#9542S), anti-cleaved PARP (1:1000, BD Pharmingen \#51-9000017), anti-caspase-3 (1:1000, CST \#9662S), anti-tubulin (1:3,000, Bio-Rad \#12004165) or anti- $\beta$-actin (1:2500, CTS \#4970S) antibody overnight at $4^{\circ} \mathrm{C}$ with rotation. Blots were then incubated in goat anti-mouse or donkey antirabbit IRDye 800CW secondary antibodies (1:10,000, LICOR \#925-32210 and \#926-32213) for $1 \mathrm{~h}$ at room temperature with rotation. Bands were detected using a ChemiDoc MP imaging system (BioRad) and quantified (Image Studio Lite, version 5.2) with normalisation to $\beta$-actin and the DMSO control per time point. Data are the average of two biological repeats unless indicated otherwise. Degradation data were plotted and fitted by nonlinear regression using a single-phase exponential decay model in GraphPad Prism.

Cell Viability Assay. MV4;11 cells were incubated with compounds at the desired concentration for $48 \mathrm{~h}$ on a clear-bottom 384-well plate. MV4;11 cells were kept in RPMI medium supplemented with 10\% FBS, Lglutamine. Initial cell density was $3 \times 10^{5}$ per $\mathrm{mL}$. The cells were treated with various concentrations of compound or $0.05 \%$ DMSO in triplicates for each concentration point. After treatment, cell viability was measured with Promega CellTiter-Glo luminescent cell viability assay kit according to the manufacturer instructions. Signal was recorded on a BMG Labtech PHERAstar luminescence plate reader with recommended settings. Data were analyzed with Graphpad Prism software to obtain $\mathrm{EC}_{50}$ values of each test compound.

Kinetic Degradation and Quantitation. HEK293 cells (ATCC) stably expressing LgBiT (Promega) were edited using CRISPR/Cas9 to endogenously HiBiT tag the N-terminal genomic loci of BRD2, BRD3, or BRD $4{ }^{31}$. For kinetic degradation assays, cells were plated in quadruplicate in white 96 -well tissue culture plates at a density of $2 \times 10^{4}$ cells per well in $100 \mu \mathrm{L}$ of growth medium and incubated overnight at $37^{\circ} \mathrm{C}$, $5 \% \mathrm{CO}_{2}$. The following day, medium was replaced with $\mathrm{CO}_{2}$-independent medium (Gibco) containing a 1:100 dilution of Endurazine (Promega) and were incubated at $37^{\circ} \mathrm{C}$ in $5 \% \mathrm{CO}_{2}$ for $2.5 \mathrm{~h}$ before addition of a 3-fold serial dilution of $1 \mu \mathrm{M}$ (SIM1-SIM3 with BRD4),10nM (SIM1 with BRD2, 3, and 4), or 100nM (ARV-771 (MedChemExpress) with BRD2, 3, and 4) final concentration of compound. Plates were placed with lids into the GloMax Discover Microplate Reader (Promega) set to $37^{\circ} \mathrm{C}$ and continuous luminescent measurements with readings every $5-15 \mathrm{~min}$ were carried out over a $24 \mathrm{~h}$ period post-compound treatment. Degradation rate and degradation plateau were calculated from above determined kinetic 
degradation profiles. Briefly, the initial degradation portion of each kinetic concentration curve was fitted to the equation:

$$
\bar{y}=\left(y_{0}-\text { plateau }\right) e^{-3 t}+\text { plateau }
$$

where $\nabla=$ degradation rate in units of $\mathrm{h}^{-1}$. The degraded fraction, Dmax, was calculated as $1-$ plateau. For each curve, the data points before onset of degradation were excluded from the fits. The Dmax was then plotted against concentration to determine $\operatorname{Dmax}_{50}$ values.

Mass spectrometry proteomics. Sample preparation. MV4;11 cells in RPMI (Invitrogen) were seeded at 5 $\times 10^{6}$ cells on a $100 \mathrm{~mm}$ plate $24 \mathrm{~h}$ before treatment. Cells were treated in triplicate by addition of test compound. After $4 \mathrm{~h}$, the cells were centrifuged at $250 \mathrm{~g}$ for $5 \mathrm{~min}$ and washed twice with $12 \mathrm{~mL}$ of cold PBS. Cells were lyzed in $500 \mu \mathrm{L}$ of $100 \mathrm{mM}$ TRIS pH 8.0, $4 \%$ (w/v) SDS supplemented with protease inhibitor cocktail (Roche). The lysate was pulse sonicated briefly and then centrifuged at $15,000 \mathrm{~g}$ for 10 min at $4^{\circ} \mathrm{C}$. Samples were quantified using a micro BCA protein assay kit (Thermo Fisher Scientific) and $200 \mu \mathrm{g}$ of each sample was processed and digested using the filter aided sample preparation method followed by alkylation with iodoacetamide and digestion with trypsin as previously described ${ }^{21}$. The samples were then desalted using a $7 \mathrm{~mm}, 3 \mathrm{~mL}$ C18 SPE cartridge column (Empore, 3M) and labeled with TMT 10-plex Isobaric Label Reagent Set (Thermo Fisher Scientific) as per the manufacturer's instructions. After labeling, the peptides from the nine samples were pooled together in equal proportion. The pooled sample was fractionated using high $\mathrm{pH}$ reverse-phase chromatography on an XBridge peptide BEH column $(130 \AA ̊$, $3.5 \mu \mathrm{m}, 2.1 \times 150 \mathrm{~mm}$, Waters) on an Ultimate 3000 HPLC system (Thermo Scientific/Dionex). Buffers $\mathrm{A}(10 \mathrm{mM}$ ammonium formate in water, $\mathrm{pH} 9)$ and $\mathrm{B}(10 \mathrm{mM}$ ammonium formate in $90 \%$ acetonitrile, $\mathrm{pH}$ 9) were used over a linear gradient of 5 to $60 \%$ buffer B over $60 \mathrm{~min}$ at a flow rate of $200 \mu \mathrm{L} \mathrm{min}^{-1}$. Then, 80 fractions were collected before concatenation into 20 fractions on the basis of the ultraviolet signal of each fraction. All the fractions were dried in a Genevac EZ-2 concentrator and resuspended in $1 \%$ formic acid for mass spectrometry analysis.

LC-MS/MS analysis. The fractions were analyzed sequentially on a Q Exactive HF Hybrid QuadrupoleOrbitrap Mass Spectrometer (Thermo Scientific) coupled to an UltiMate 3000 RSLCnano ultra HPLC system (Thermo Scientific) and EasySpray column $(75 \mu \mathrm{m} \times 50 \mathrm{~cm}$, PepMap RSLC C18 column, $2 \mu \mathrm{m}$, $100 \AA$, Thermo Scientific). Buffers A ( $0.1 \%$ formic acid in water) and B $(0.08 \%$ formic acid in $80 \%$ acetonitrile) were used over a linear gradient from 5 to $35 \%$ buffer $B$ over $125 \mathrm{~min}$ at $300 \mathrm{~nL} \mathrm{~min}{ }^{-1}$. The column temperature was $50^{\circ} \mathrm{C}$. The mass spectrometer was operated in data dependent mode with a single mass spectrometry survey scan from $335-1,600 \mathrm{~m} / \mathrm{z}$ followed by 15 sequential $\mathrm{m} / \mathrm{z}$ dependent MS2 scans. The 15 most intense precursor ions were sequentially fragmented by higher energy collision dissociation. The MS1 isolation window was set to $0.7 \mathrm{~m} / \mathrm{z}$ and the resolution set at 120,000 . MS2 resolution was set at 60,000. The automatic gain control (AGC) targets for MS1 and MS2 were set at $3 \times$ $10^{6}$ ions and $1 \times 10^{5}$ ions, respectively. The normalized collision energy was set at $32 \%$. The maximum ion injection times for MS1 and MS2 were set at 50 and $200 \mathrm{~ms}$, respectively. 
Peptide and protein identification. The raw mass spectrometry data files for all 20 fractions were merged and searched against the Uniprot-sprot-Human-Canonical database by MaxQuant software v.1.6.0.16 for protein identification and TMT reporter ion quantitation. The MaxQuant parameters were set as follows: enzyme used trypsin/P; maximum number of missed cleavages equal to two; precursor mass tolerance equal to $10 \mathrm{ppm}$; fragment mass tolerance equal to $20 \mathrm{ppm}$; variable modifications: oxidation $(\mathrm{M})$, acetyl (N-term), deamidation (NQ), Gln $\rightarrow$ pyro-Glu (Q N-term); fixed modifications: carbamidomethyl (C). The data was filtered by applying a $1 \%$ false discovery rate followed by exclusion of proteins with fewer than two unique peptides. Quantified proteins were filtered if the absolute fold-change difference between the three DMSO replicates was $\geq 1.5$.

Monitoring cMyc Loss and Cell Viability in MV4;11 Cells. CRISPR cMyc-HiBiT MV4;11 cells (Promega) were plated at a density of $5 \times 10^{4}$ cells per well in solid, white 96-well tissue culture plates (Corning Costar \#3917). Following an overnight incubation, they were treated with 1-100nM concentration of the indicated compounds and at the plotted time points, cMyc levels were determined using luminescent measurement with NanoGlo HiBiT lytic reagent (Promega). Replicate plates of all compound treatments were prepared and at identical timepoints as the protein level measurements, cell viability was measured using Cell-Titer Glo (Promega). Plates were shaken on an orbital shaker for 10-20 min before reading luminescence on a GloMax Discover Microplate Reader (Promega).

Caspase-Glo@ $3 / 7$ assays. 22Rv1 cells were seeded at 10,000 cells/well of white 96 well plates (Corning \#3917) 12-24 h before treatment with test compounds with and without inhibitors or an equivalent volume of DMSO for $24 \mathrm{~h} .100 \mu \mathrm{L} /$ well of Caspase-Glo 3/7 Reagent (Promega) was added and the plate shaken at $500 \mathrm{rpm}$ for $30 \mathrm{~s}$. The plate was incubated for $2 \mathrm{~h}$ and luminescence measured using a PHERAstar FS plate reader (BMG Labtech).

Clonogenic assay. 22Rv1 cells were treated with 10nM SIM1, cis-SIM1, MT1, MZ1 and ARV-711 for $24 \mathrm{~h}$. The next day, cells were trypsinised and counted. 500 cells were re-plated and allowed to grow at $37^{\circ} \mathrm{C}$ and $5 \% \mathrm{CO}_{2}$ for 20 days. After 20 days incubation, the cells were fixed with ice-cold $100 \%(\mathrm{v} / \mathrm{v})$ methanol for $30 \mathrm{~min}$ at $4^{\circ} \mathrm{C}$. Afterwards, methanol was removed, and the cells were stained with $500 \mu \mathrm{l} 0.1 \%$ crystalviolet dye (in $\mathrm{MeOH}$ ) for 30 min at room temperature. Following incubation, the cells were washed with $\mathrm{dH}_{2} \mathrm{O}$ and left to dry overnight. Plates were scanned on an Epson Perfection V800 Photo scanner. And image analysis was done in ImageJ software. Plating efficiency (PE) was calculated by counting colonies for each treatment condition and dividing the average by number of cells plated. Survival fraction was determined by diving PE of treated cells by PE of untreated cells, multiplied by $100{ }^{59}$. Bar graphs were generated using GraphPad Prism software. Error bars indicate the mean values \pm S.D. Two independent experiments were performed.

Protein expression and purification. For expression of BRD4 tandem construct, N-terminally His6-tagged BRD4 (1-463) or similar mutants were expressed in Escherichia coli BL21(DE3) at $18^{\circ} \mathrm{C}$ for $16 \mathrm{~h}$ using $0.4 \mathrm{mM}$ isopropyl $\beta$-D-1-thiogalactopyranoside (IPTG). E. coli cells were lysed using a pressure cell homogenizer (Stansted Fluid Power) and lysate clarified by centrifugation. His6-tagged VCB was purified 
on a HisTrap HP affinity column (GE Healthcare) by elution with an imidazole gradient. The His6 tag was removed using TEV protease and the untagged complex dialyzed into low-concentration imidazole buffer. BRD4 was then flowed through the HisTrap HP column a second time, allowing impurities to bind, as the complex eluted without binding. BRD4 was then additionally purified by anion exchange and sizeexclusion chromatography using Mono $S$ and Superdex-200 columns (GE Healthcare), respectively. The final purified complex was stored in $20 \mathrm{mM}$ HEPES, $\mathrm{pH} 7.5,100 \mathrm{mM}$ sodium chloride and $1 \mathrm{mM}$ TCEP. The VCB complex was expressed and purified as described previously ${ }^{21}$. Briefly, N-terminally His6-tagged VHL (54-213), ElonginC (17-112) and ElonginB (1-104) were co-expressed and the complex was isolated by Ni-affinity chromatography, the His6 tag was removed using TEV protease, and the complex further purified by anion exchange and size-exclusion chromatography. The BET protein BDs were expressed and purified as described previously ${ }^{21}$. Briefly, N-terminally His6-tagged BRD2-BD1 (71-194), BRD2-BD2 (344-455), BRD3-BD1 (24-146), BRD3-BD2 (306-416), BRD4-BD1 (44-178) and BRD4-BD2 (333-460) were expressed and isolated by Ni-affinity chromatography and size-exclusion chromatography.

Size exclusion chromatography (SEC). SEC experiments were carried out in a ÄKTA pure system (GE Healthcare) at room temperature. The oligomeric state of the BRD4 BD1-BD2 tandem protein in solution was analyzed by gel filtration in a buffer containing $20 \mathrm{mM} \mathrm{HEPES} \mathrm{(pH} \mathrm{7.5),} 100 \mathrm{mM} \mathrm{NaCl}$ and $1 \mathrm{mM}$ TCEP using a Superdex 200 Increase 10/300 GL column (GE Healthcare) calibrated with globular proteins of known molecular weight (GE Healthcare, 28-4038-41/42). BRD4 tandem $(25 \mu \mathrm{M})$ was incubated with SIM1 $(25 \mu \mathrm{M}), \mathrm{MZ1}(25 \mu \mathrm{M}), \mathrm{MT} 1(25 \mu \mathrm{M})$ or DMSO $(0.5 \%)$ for $30 \mathrm{~min}$ at room temperature prior to injection. Sample volume for each injection was $200 \mu \mathrm{l}$, and the flow rate was $0.8 \mathrm{ml} / \mathrm{min}$. Peak elution was monitored using ultraviolet absorbance at $280 \mathrm{~nm}$.

ITC. Titrations were performed as reverse titration on an ITC200 micro-calorimeter (Malvern). SIM1 was not soluble enough to be loaded at the required concentrations in the syringe (normal direct titration), therefore reverse titrations were performed. The titrations consisted of 19 injections of $2 \mu$ landem BRD 4 BD1-BD2 construct (WT or N140F or N433F) solution in $20 \mathrm{mM}$ Bis-Tris propane, $100 \mathrm{mM} \mathrm{NaCl}, 1 \mathrm{mM}$ TCEP, $1.6 \%$ DMSO, pH 7.5, at a rate of $0.5 \mu \mathrm{l} / \mathrm{s}$ at $120 \mathrm{~s}$ time intervals. An initial injection of protein $(0.4 \mu \mathrm{l})$ was made and discarded during data analysis. All experiments were performed at $25^{\circ} \mathrm{C}$, whilst stirring at 750 r.p.m. SIM1 from $10 \mathrm{mM}$ DMSO stock solution and VCB were diluted in buffer containing $20 \mathrm{mM}$ BisTris propane, $100 \mathrm{mM} \mathrm{NaCl}, 1 \mathrm{mM}$ TCEP, $\mathrm{pH} 7.5$. The final DMSO concentration was $1.6 \% \mathrm{v} / \mathrm{v}$. BRD4 protein $(200 \mu \mathrm{M}$, in the syringe) was titrated into the SIM1-VCB complex (SIM1 $16 \mu \mathrm{M}$, VCB $32 \mu \mathrm{M}$, in the cell). Data were fitted to a single-binding site model for each BRD4 mutant to obtain the stoichiometry (n), the dissociation constant $(\mathrm{Kd})$ and the enthalpy of binding $(\Delta \mathrm{H})$. Data for WT BRD4 was fitted to a two sets of sites model to account for the reverse titration set-up whereby a two-site protein is titrated into a bivalent ligand (see Malvern MicroCal ITC analysis software using Origin $^{\text {TM }}$

User Manual, pg. 102). Data fitting was performed using Microcal LLC ITC200 Origin software provided by the manufacturer. 
AlphaLISA assays. Ligands were titrated against 4nM His-tagged BRD4 BD2 and 10nM biotinylated JQ1. All reagents were diluted in $50 \mathrm{mM}$ HEPES, $100 \mathrm{mM} \mathrm{NaCl}, 0.1 \% \mathrm{BSA}, 0.02 \% \mathrm{CHAPS}, \mathrm{pH} 7.5$ (final

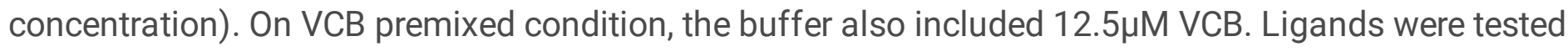
over an 11-point 3-fold serial dilution in duplicates for each concentration point, starting at $100 \mu \mathrm{M}$ without VCB or starting at $10 \mu \mathrm{M}$ with $20 \mu \mathrm{M}$ VCB, and giving a final DMSO concentration of $1 \%$. Binding was detected using anti-His6 antibody-conjugated AlphaLISA acceptor beads and streptavidin-coated donor beads (PerkinElmer), with a final concentration of $10 \mu \mathrm{g} / \mathrm{ml}$ for each bead). Titrations were prepared in white 384-well Alphaplates (PerkinElmer), and read on a Pherastar FS plate reader (BMG) equipped with an AlphaLISA excitation/emission module. Data was analyzed and dose-response curves generated using GraphPad Prism 7. Each assay well had a final volume of $25 \mu$ l. First $10 \mu \mathrm{l}$ of $2.5 \mathrm{X}$ ligand or 2.5X ligand with VCB was mixed with $5 \mu \mathrm{l}$ of a $5 \mathrm{X}$ mix of bromodomain and biotinylated JQ1 and incubated for $1 \mathrm{~h}$ at room temperature. The assay plate was then moved to a dark room and $5 \mu \mathrm{l}$ of $5 \mathrm{X}$ acceptor beads were added and incubated for $1 \mathrm{~h}$. Then (still in darkness) $5 \mu \mathrm{l}$ of $5 \mathrm{X}$ donor beads were added, the plate was incubated for $1 \mathrm{~h}$ before being read.

Fluorescence polarization assay. Fluorescence polarization (FP) competitive binding assays were run as described previously ${ }^{33}$ with a final volume of $15 \mu \mathrm{L}$, with each well solution containing $15 \mathrm{nM}$ VCB protein, 10nM FAM-labeled HIF-1 a peptide (FAM-DEALAHypYIPMDDDFQLRSF, "JC9") and decreasing concentrations of PROTAC (14-point 2-fold serial dilution starting from 50 $\mu \mathrm{M}$ ) or PROTAC:BRD4 tandem

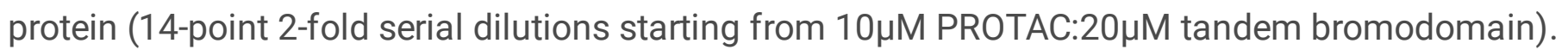
Assays were prepared in triplicate on 384-well plates (Corning 3575) and all measurements taken using a PHERAstar FS (BMG LABTECH) with fluorescence excitation and emission wavelengths $(\lambda)$ of 485 and $520 \mathrm{~nm}$, respectively. Components were dissolved from stock solutions using $100 \mathrm{mM}$ Bis-Tris propane, $100 \mathrm{mM} \mathrm{NaCl}, 1 \mathrm{mM}$ TCEP, $\mathrm{pH} 7.5$, and DMSO was added as appropriate to ensure a final concentration of $1 \%$. Control wells containing VCB and JC9 with no compound (zero displacement), or JC9 in the absence of protein (maximum displacement) were also included to allow for normalization. Normalized (\%) displacement values were plotted against log[PROTAC] and curves were fitted by nonlinear regression using GraphPad Prism to determine the $\mathrm{IC}_{50}$ values for each titration. $K_{i}$ values were back-calculated from the $K_{\mathrm{d}}$ for JC9 ( 2nM, determined from direct binding) and fitted $\mathrm{IC}_{50}$ values, as described previously ${ }^{60}$. Cooperativity (a) values were calculated from the ratio of binary $K_{\mathrm{i}}$ and ternary $K_{\mathrm{i}}$ values determined for JC9 displacement by SIM1 alone or SIM1 + BRD4, respectively.

SPR binding studies. SPR experiments were performed on Biacore T200 instruments (GE Healthcare) as described previously ${ }^{33}$. Immobilization of Biotinylated VCB was carried out at $25^{\circ} \mathrm{C}$ on a pre-coupled Series S SA chip in running buffer containing $20 \mathrm{mM}$ TRIS, $150 \mathrm{mM}$ potassium chloride, $2 \mathrm{mM}$ magnesium chloride, $2 \mathrm{mM}$ TCEP, $0.005 \%$ TWEEN 20, 1\% DMSO; pH 8.3. Multiple surface densities of biotinylated VHL were used (40, 80 and $120 \mathrm{RU})$. Biotinylated VCB was prepared as previously described ${ }^{33}$. All interaction experiments were performed at $9^{\circ} \mathrm{C}$ in running buffer containing $20 \mathrm{mM}$ TRIS, $150 \mathrm{mM}$ potassium chloride, $2 \mathrm{mM}$ magnesium chloride, $2 \mathrm{mM}$ TCEP, $0.005 \%$ TWEEN 20, 1\% DMSO; pH 8.3. SIM1 (10mM in $100 \%$ DMSO) were initially prepared at $1 \mu \mathrm{M}$ in running buffer with a concentration of $2 \%$ DMSO. This

Page 19/45 
solution was mixed 1:1 with a solution of $50 \mu \mathrm{M}$ of the BRD4 tandem protein in running buffer without

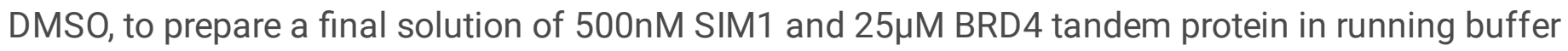
containing $1 \%$ DMSO. This complex was then serially diluted in running buffer containing $2 \mu \mathrm{M}$ bromodomain and 1\% DMSO (5-point five-fold serial dilution). Solutions were injected sequentially in single-cycle kinetic format without regeneration (three replicate series per experimental repeat, contact time $100 \mathrm{sec}$, flow rate $100 \mu \mathrm{L} / \mathrm{min}$, dissociation time $800 \mathrm{sec}$ ) using a stabilization period of $30 \mathrm{sec}$ and syringe wash ( $50 \%$ DMSO) between injections. High flow rates and multiple surface densities were used to minimise mass transfer effects. Two series of blank injections were performed for all single cycle experiments. Sensorgrams from reference surfaces and blank injections were subtracted from the raw data before data analysis using Biacore insight evaluation software. To calculate the association rate $\left(k_{o n}\right)$, dissociation rate $\left(k_{\text {off }}\right)$, and dissociation constant $\left(K_{D}\right)$, experiments were fitted using a 1:1 Langmuir interaction model, with a term for mass-transport included.

\section{NanoBRET Ubiquitination, Ternary Complex, and Biosensor Experiments.}

For endogenous live cell BET:Ubiquitin and BET:VHL assays, CRISPR HiBiT-BRD2, HiBiT-BRD3, and HiBiTBRD4 HEK293 cells stably expressing LgBiT were transfected with $2 \mu \mathrm{g}$ of HaloTag-UBB or HaloTag-VHL vectors in 6-well plates using FuGENE HD (Promega). For full transient NanoBRET experiments with NanoLuc-BRD4 WT, N433F, or N140F mutants, HEK293 cells $\left(8 \times 10^{5}\right)$ were co-transfected with $0.02 \mu \mathrm{g}$ NanoLuc-BRD4 and $2 \mu \mathrm{g}$ of HaloTag-VHL vectors. For transient NanoBRET experiments with the BRD4 NLBD1-BD2-HT biosensor containing either the WT tandem BD1-BD2 domain the N433F mutation, HEK293 cells $\left(8 \times 10^{5}\right)$ were transfected with $0.02 \mu \mathrm{g}$ biosensor plasmid and $2 \mu \mathrm{g}$ carrier DNA. The following day, transfected cells $\left(2 \times 10^{4}\right)$ were replated in quadruplicate into white 96 -well tissue culture plates in the presence or absence of HaloTag NanoBRET 618 Ligand (Promega) and incubated overnight at $37^{\circ} \mathrm{C}, 5 \%$ $\mathrm{CO}_{2}$. For kinetic experiments, medium was replaced with Opti-MEM (Gibco) containing a 1:100 dilution of Vivazine (Promega), and plates were incubated at $37^{\circ} \mathrm{C}, 5 \% \mathrm{CO}_{2}$, for $1 \mathrm{~h}$ before addition of DMSO or $10 \mathrm{nM}-1 \mu \mathrm{M}$ final concentration of the indicated compounds. Continual BRET measurements were then made every 3 min up to $5 \mathrm{~h}$ on a CLARIOstar equipped with an atmospheric control unit (BMG Labtech) set to $37^{\circ} \mathrm{C}$ and $5 \% \mathrm{CO}_{2}$. For the biosensor experiments, the cells were treated with a 3-fold serial titration of $10 \mu \mathrm{M}$ of the indicated compounds. NanoBRET NanoGlo (Promega) substrate was added and BRET was measured two hours post-compound treatment using a GloMax Discover Microplate Reader (Promega). Dual filtered luminescence was collected with a 460/80nm bandpass filter and a $610 \mathrm{~nm}$ long pass filter (acceptor, HaloTag NanoBRET ligand) using an integration time of $0.5 \mathrm{~s}$. For all NanoBRET experiments, background subtracted NanoBRET ratios expressed in milliBRET units were calculated from the equation:

mBRET ratio $=\left(\frac{\text { acceptor channel }}{\text { donor channel }}-\frac{\text { acceptor channel (no ligand) }}{\text { donor channel (no ligand) }}\right) 1000$.

Fold increase in BRET was calculated by normalizing $\mathrm{MBRET}$ ratios to the average $\mathrm{mBRET}$ ratios for DMSO controls. 
NanoBRET Target Engagement and Residence Time. For target engagement experiments in live and permeabilized cells, CRISPR HiBiT-BRD4 HEK293 cells stably expressing LgBiT were plated into white 96well tissue culture plates at a density of $2 \times 10^{4}$ cells/well. Cells were equilibrated for $1 \mathrm{~h}$ with energy transfer probes and the indicated test compound prior to NanoBRET measurements. NanoBRET tracers were prepared at a working concentration of 20X in tracer dilution buffer (12.5mM HEPES, 31.25\% PEG$400, \mathrm{pH} 7.5$ ). NanoBRET BRD Tracer- 02 was added to cells at a final concentration of $0.5 \mu \mathrm{M}$. To measure NanoBRET in live cells, NanoBRET NanoGlo Substrate and Extracellular NanoLuc Inhibitor (Promega) were added according to the manufacturer's recommended protocol, and filtered luminescence was measured on a GloMax Discover Microplate Reader (Promega) equipped with $450 \mathrm{~nm}$ BP filter (donor) and $600 \mathrm{~nm}$ LP filter (acceptor) using $0.3 \mathrm{~s}$ integration time. To measure NanoBRET in permeabilized cells, digitonin was added to the cells to a final concentration of $50 \mu \mathrm{g} / \mathrm{mL}$ and Extracellular NanoLuc inhibitor was omitted during the detection step. For residence time experiments CRISPR HiBiT-BRD4 HEK293 cells stably expressing LgBiT were trypsinized, washed and resuspended to a density of $2 \times 10^{5}$ cells/ml in Opti-MEM and incubated with either $1 \mu \mathrm{M}$ JQ1, $1 \mu \mathrm{M}$ SIM1, 100nM cis-SIM1, 100nM MT1, or $10 \mu \mathrm{M}$ MZ1 representing the representative IC80 values for tracer displacement in live cell format. Cells were incubated in $15 \mathrm{~mL}$ conical tubes with caps loosened in a tissue culture incubator for $1 \mathrm{~h}$. Following incubation, cells were spun at 300x $g$ for 5 min, washed once with Opti-MEM, spun a second time at 300x $g$ for $5 \mathrm{~min}$, then resuspended with fresh Opti-MEM before plating at $2 \times 10^{4}$ cells/well. NanoBRET BRD Tracer-02 was added at a final concentration of $0.5 \mu \mathrm{M}$ cells and kinetic NanoBRET measurements were collected. NanoBRET ratios were expressed in milliBRET units and calculated according to the equation in the NanoBRET Ubiquitination, Ternary Complex and Biosensor Experiments section.

\section{Declarations}

\section{ORCID IDs:}

Alessio Ciulli: 0000-0002-8654-1670

Danette L. Daniels: 0000-0002-7659-3020

Scott Hughes: 0000-0001-8360-929X

Chiara Maniaci: 0000-0001-7887-7388

Andrea Testa: 0000-0002-8973-9711

\section{Funding Sources}

Research reported in this publication was supported by the European Research Council (ERC, Starting Grant ERC-2012-StG-311460 DrugE3CRLs to A.C.), and Ono Pharma (visiting scientist fund to S.I.). Biophysics and drug discovery activities were supported by Wellcome Trust strategic awards to Dundee 
(100476/Z/12/Z and 094090/Z/10/Z, respectively). K.M.R., S.D.M., N.M., M.U., and D.L.D. are employees of Promega Corporation.

\section{Competing Interests}

The authors declare the following competing financial interest(s): The Ciulli laboratory receives or has received sponsored research support from Almirall, Amphista therapeutics, Boehringer Ingelheim, Eisai, Nurix therapeutics, and Ono Pharmaceuticals. A.C. is a scientific founder, shareholder, non-executive director and consultant of Amphista therapeutics, a company that is developing targeted protein degradation therapeutic platforms. Promega Corporation is the commercial owner by assignment of patents of the HaloTag, NanoLuc, NanoBRET target engagement, and HiBiT technologies and their applications.

\section{ACKNOWLEDGMENTS}

We thank Adam Bond for the synthesis of ARV-771, and also Will Farnaby and Thomas Kirkland for discussions and critical reading of the manuscript.

\section{Author contributions}

A.C. and D.L.D. conceived the idea, directed and supervised the project, and have overall project responsibility. S.I. optimized synthetic routes and synthesized all compounds, performed degradation and cell viability assays, expressed and purified proteins, and performed SEC, AlphaLISA, ITC and SPR assays. K.M.R. performed kinetic degradation assays, and NanoBRET cellular ternary complex, biosensor, ubiquitination, and residence time assays. V.V. performed PARP-cleavage and clonogenic assays. C.W. performed degradation and Caspase-Glo assays. S.J.H. performed FP displacement assays. N.T. performed mass-spec proteomics assays. S.D.M. performed cellular cMyc and viability assays. N.M. performed the NanoBRET biosensor and mutant ternary complex assays. K.-H. C. designed and cloned tandem BRD4 WT and mutant constructs. A.T., C.M. and A.C. designed compounds. A.T. and C.M. designed synthetic routes. M.U. discussed strategy and analyzed data. S.I., K.M.R, D.L.D. and A.C. wrote the manuscript with contributions from all authors.

\section{References}

1. Deshaies, R. J. Multispecific drugs herald a new era of biopharmaceutical innovation. Nature 580, 329-338 (2020).

2. Che, Y., Gilbert, A. M., Shanmugasundaram, V. \& Noe, M. C. Inducing protein-protein interactions with molecular glues. Bioorg Med Chem Lett 28, 2585-2592 (2018).

3. Maniaci, C. \& Ciulli, A. Bifunctional chemical probes inducing protein-protein interactions. Curr Opin Chem Biol 52, 145-156 (2019).

4. Pettersson, M. \& Crews, C. M. PROteolysis TArgeting Chimeras (PROTACs) - Past, present and future. Drug Discovery Today: Technologies 31, 15-27 (2019). 
5. Petzold, G., Fischer, E. S. \& Thomä, N. H. Structural basis of lenalidomide-induced CK1a degradation by the CRL4(CRBN) ubiquitin ligase. Nature 532, 127-130 (2016).

6. Matyskiela, M. E. et al. Crystal structure of the SALL4-pomalidomide-cereblon-DDB1 complex. Nat Struct Mol Biol 27, 319-322 (2020).

7. Bussiere, D. E. et al. Structural basis of indisulam-mediated RBM39 recruitment to DCAF15 E3 ligase complex. Nat Chem Biol 16, 15-23 (2020).

8. Słabicki, M. et al. The CDK inhibitor CR8 acts as a molecular glue degrader that depletes cyclin K. Nature 585, 293-297 (2020).

9. Mayor-Ruiz, C. et al. Rational discovery of molecular glue degraders via scalable chemical profiling. Nat Chem Bio/ 348, 1376 (2020).

10. Zengerle, M., Chan, K.-H. \& Ciulli, A. Selective Small Molecule Induced Degradation of the BET Bromodomain Protein BRD4. ACS Chem Biol 10, 1770-1777 (2015).

11. Winter, G. E. et al. Phthalimide conjugation as a strategy for in vivo target protein degradation. Science 348, 1376-1381 (2015).

12. Bondeson, D. P. et al. Catalytic in vivo protein knockdown by small-molecule PROTACs. Nat Chem Biol 11, 611-617 (2015).

13. Salami, J. et al. Androgen receptor degradation by the proteolysis-targeting chimera ARCC-4 outperforms enzalutamide in cellular models of prostate cancer drug resistance. Commun Bio/ 1, 100 (2018).

14. Hu, J. et al. Discovery of ERD-308 as a Highly Potent Proteolysis Targeting Chimera (PROTAC) Degrader of Estrogen Receptor (ER). J. Med. Chem. 62, 1420-1442 (2019).

15. Qin, C. et al. Discovery of QCA570 as an Exceptionally Potent and Efficacious Proteolysis Targeting Chimera (PROTAC) Degrader of the Bromodomain and Extra-Terminal (BET) Proteins Capable of Inducing Complete and Durable Tumor Regression. J. Med. Chem. 61, 6685-6704 (2018).

16. Farnaby, W. et al. BAF complex vulnerabilities in cancer demonstrated via structure-based PROTAC design. Nat Chem Biol 15, 672-680 (2019).

17. Zorba, A. et al. Delineating the role of cooperativity in the design of potent PROTACs for BTK. P NatI Acad Sci Usa 115, E7285-E7292 (2018).

18. Popow, J. et al. Highly Selective PTK2 Proteolysis Targeting Chimeras to Probe Focal Adhesion Kinase Scaffolding Functions. J. Med. Chem. 62, 2508-2520 (2019).

19. Burslem, G. M. et al. The Advantages of Targeted Protein Degradation Over Inhibition: An RTK Case Study. Cell Chem Biol 25, 67-77.e3 (2018).

20. Bensimon, A. et al. Targeted Degradation of SLC Transporters Reveals Amenability of Multi-Pass Transmembrane Proteins to Ligand-Induced Proteolysis. Cell Chem Bio/ 27, 728-739.e9 (2020).

21. Gadd, M. S. et al. Structural basis of PROTAC cooperative recognition for selective protein degradation. Nat Chem Biol 13, 514-521 (2017). 
22. Bondeson, D. P. et al. Lessons in PROTAC Design from Selective Degradation with a Promiscuous Warhead. Cell Chem Biol 25, 78-87.e5 (2018).

23. Olson, C. M. et al. Pharmacological perturbation of CDK9 using selective CDK9 inhibition or degradation. Nat Chem Biol 14, 163-170 (2018).

24. Tovell, H. et al. Design and Characterization of SGK3-PROTAC1, an Isoform Specific SGK3 Kinase PROTAC Degrader. ACS Chem Biol 14, 2024-2034 (2019).

25. Testa, A. et al. 3-Fluoro-4-hydroxyprolines: Synthesis, Conformational Analysis, and Stereoselective Recognition by the VHL E3 Ubiquitin Ligase for Targeted Protein Degradation. J Am Chem Soc 140, 9299-9313 (2018).

26. Han, X. et al. Discovery of Highly Potent and Efficient PROTAC Degraders of Androgen Receptor (AR) by Employing Weak Binding Affinity VHL E3 Ligase Ligands. J. Med. Chem. 62, 11218-11231 (2019).

27. Maniaci, C. et al. Homo-PROTACs: bivalent small-molecule dimerizers of the VHL E3 ubiquitin ligase to induce self-degradation. Nat Commun 8, 830 (2017).

28. Potjewyd, F. et al. Degradation of Polycomb Repressive Complex 2 with an EED-Targeted Bivalent Chemical Degrader. Cell Chem Biol 27, 47-56.e15 (2020).

29. Zoppi, V. et al. Iterative Design and Optimization of Initially Inactive Proteolysis Targeting Chimeras (PROTACs) Identify VZ185 as a Potent, Fast, and Selective von Hippel-Lindau (VHL) Based Dual Degrader Probe of BRD9 and BRD7. J. Med. Chem. 62, 699-726 (2019).

30. Crew, A. P. et al. Identification and Characterization of von Hippel-Lindau-Recruiting Proteolysis Targeting Chimeras (PROTACs) of TANK-Binding Kinase 1. J. Med. Chem. 61, 583-598 (2018).

31. Riching, K. M. et al. Quantitative Live-Cell Kinetic Degradation and Mechanistic Profiling of PROTAC Mode of Action. ACS Chem Biol 13, 2758-2770 (2018).

32. Daniels, D. L., Riching, K. M. \& Urh, M. Monitoring and deciphering protein degradation pathways inside cells. Drug Discovery Today: Technologies 31, 61-68 (2019).

33. Roy, M. J. et al. SPR-Measured Dissociation Kinetics of PROTAC Ternary Complexes Influence Target Degradation Rate. ACS Chem Biol 14, 361-368 (2019).

34. Nowak, R. P. et al. Plasticity in binding confers selectivity in ligand-induced protein degradation article. Nat Chem Bio/ 14, 706-714 (2018).

35. Mammen, M., Choi, S.-K. \& Whitesides, G. M. Polyvalent Interactions in Biological Systems: Implications for Design and Use of Multivalent Ligands and Inhibitors. Angew Chem Int Ed Eng/ 37, 2754-2794 (1998).

36. Kiessling, L. L., Gestwicki, J. E. \& Strong, L. E. Synthetic multivalent ligands as probes of signal transduction. Angew Chem Int Ed Eng/ 45, 2348-2368 (2006).

37. Wu, Q. et al. A chemical toolbox for the study of bromodomains and epigenetic signaling. Nat Commun 10, 1915-14 (2019). 
38. Gilan, O. et al. Selective targeting of BD1 and BD2 of the BET proteins in cancer and immunoinflammation. Science 368, 387-394 (2020).

39. Faivre, E. J. et al. Selective inhibition of the BD2 bromodomain of BET proteins in prostate cancer. Nature 1-19 (2020). doi:10.1038/s41586-020-1930-8

40. Raina, K. et al. PROTAC-induced BET protein degradation as a therapy for castration-resistant prostate cancer. $P$ Natl Acad Sci Usa 113, 7124-7129 (2016).

41. Winter, G. E. et al. BET Bromodomain Proteins Function as Master Transcription Elongation Factors Independent of CDK9 Recruitment. Mol Cell 67, 5-18.e19 (2017).

42. Tanaka, M. et al. Design and characterization of bivalent BET inhibitors. Nat Chem Bio/ 12, 10891096 (2016).

43. Waring, M. J. et al. Potent and selective bivalent inhibitors of BET bromodomains. Nat Chem Bio/ 12, 1097-1104 (2016).

44. Frost, J. et al. Potent and selective chemical probe of hypoxic signalling downstream of HIF-a hydroxylation via VHL inhibition. Nat Commun 7, 13312 (2016).

45. Zhu, X. F. et al. Knockdown of heme oxygenase-1 promotes apoptosis and autophagy and enhances the cytotoxicity of doxorubicin in breast cancer cells. Oncol Lett 10, 2974-2980 (2015).

46. Runcie, A. C. et al. Optimization of a 'bump-and-hole' approach to allele-selective BET bromodomain inhibition. Chem Sci 9, 2452-2468 (2018).

47. Smith, B. E. et al. Differential PROTAC substrate specificity dictated by orientation of recruited E3 ligase. Nat Commun 10, 131 (2019).

48. Hughes, S. J. \& Ciulli, A. Molecular recognition of ternary complexes: a new dimension in the structure-guided design of chemical degraders. Essays Biochem 61, 505-516 (2017).

49. Fisher, S. L. \& Phillips, A. J. Targeted protein degradation and the enzymology of degraders. Curr Opin Chem Biol 44, 47-55 (2018).

50. Ottis, P. et al. Cellular Resistance Mechanisms to Targeted Protein Degradation Converge Toward Impairment of the Engaged Ubiquitin Transfer Pathway. ACS Chem Biol 14, 2215-2223 (2019).

51. Mayor-Ruiz, C. et al. Plasticity of the Cullin-RING Ligase Repertoire Shapes Sensitivity to LigandInduced Protein Degradation. Mol Cell 75, 849-858.e8 (2019).

52. Zhang, L., Riley-Gillis, B., Vijay, P. \& Shen, Y. Acquired Resistance to BET-PROTACs (ProteolysisTargeting Chimeras) Caused by Genomic Alterations in Core Components of E3 Ligase Complexes. Mol Cancer Ther 18, 1302-1311 (2019).

53. Ruthenburg, A. J., Li, H., Patel, D. J. \& Allis, C. D. Multivalent engagement of chromatin modifications by linked binding modules. Nat Rev Mol Cell Bio/ 8, 983-994 (2007).

54. Sun, Q. et al. Discovery of small molecules that bind to K-Ras and inhibit Sos-mediated activation. Angew Chem Int Ed Eng/ 51, 6140-6143 (2012).

55. Lucas, X., Van Molle, I. \& Ciulli, A. Surface Probing by Fragment-Based Screening and Computational Methods Identifies Ligandable Pockets on the von Hippel-Lindau (VHL) E3 Ubiquitin Ligase. J. Med. 
Chem. 61, 7387-7393 (2018).

56. Yamazoe, S. et al. Heterobifunctional Molecules Induce Dephosphorylation of Kinases-A Proof of Concept Study. J. Med. Chem. 63, 2807-2813 (2020).

57. Siriwardena, S. U. et al. Phosphorylation-Inducing Chimeric Small Molecules. J Am Chem Soc 142, 14052-14057 (2020).

58. Banik, S. M. et al. Lysosome-targeting chimaeras for degradation of extracellular proteins. Nature 584, 291-297 (2020).

59. Munshi, A., Hobbs, M. \& Meyn, R. E. Clonogenic cell survival assay. Methods Mol Med 110, 21-28 (2005).

60. Van Molle, I. et al. Dissecting fragment-based lead discovery at the von Hippel-Lindau protein:hypoxia inducible factor 1a protein-protein interface. Chem Bio/ 19, 1300-1312 (2012).

\section{Figures}


a
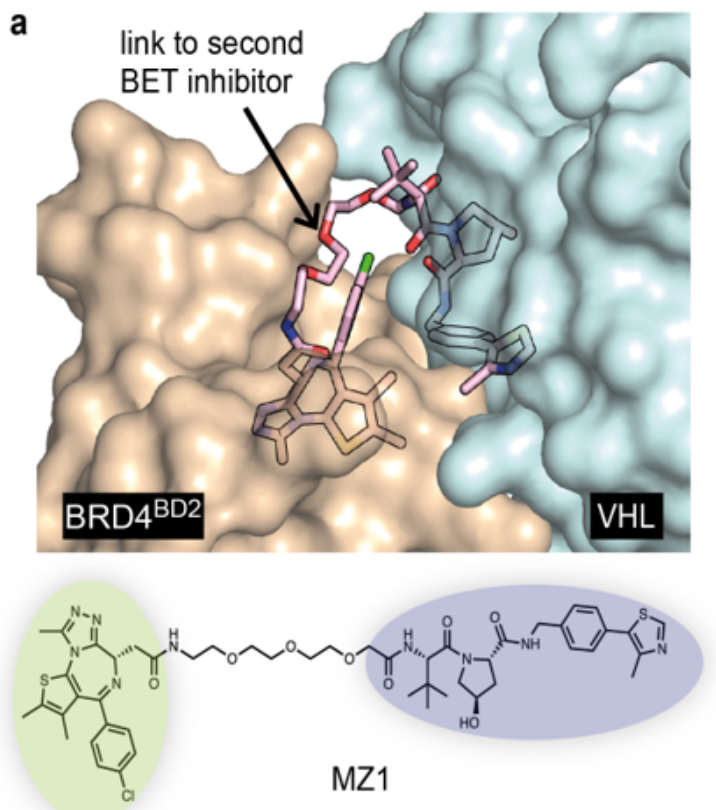

C

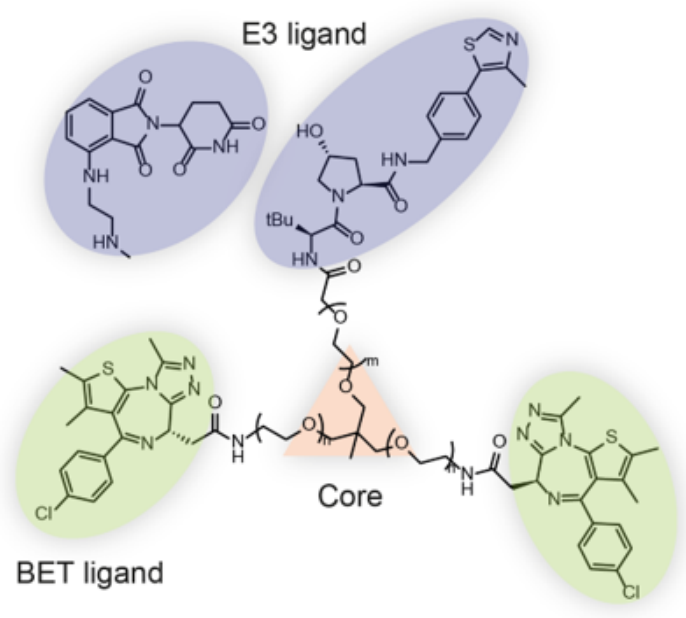

\begin{tabular}{|cccc|}
\hline & $n$ & $m$ & $\begin{array}{c}\text { E3 } \\
\text { ligand }\end{array}$ \\
\hline SIM1 & 3 & 0 & VHL \\
\hline SIM2 & 3 & 1 & VHL \\
\hline SIM3 & 4 & 0 & VHL \\
\hline SIM4 & 3 & 0 & CRBN \\
\hline SIM5 & 3 & 1 & CRBN \\
\hline SIM6 & 4 & 0 & CRBN \\
\hline
\end{tabular}

\section{b}
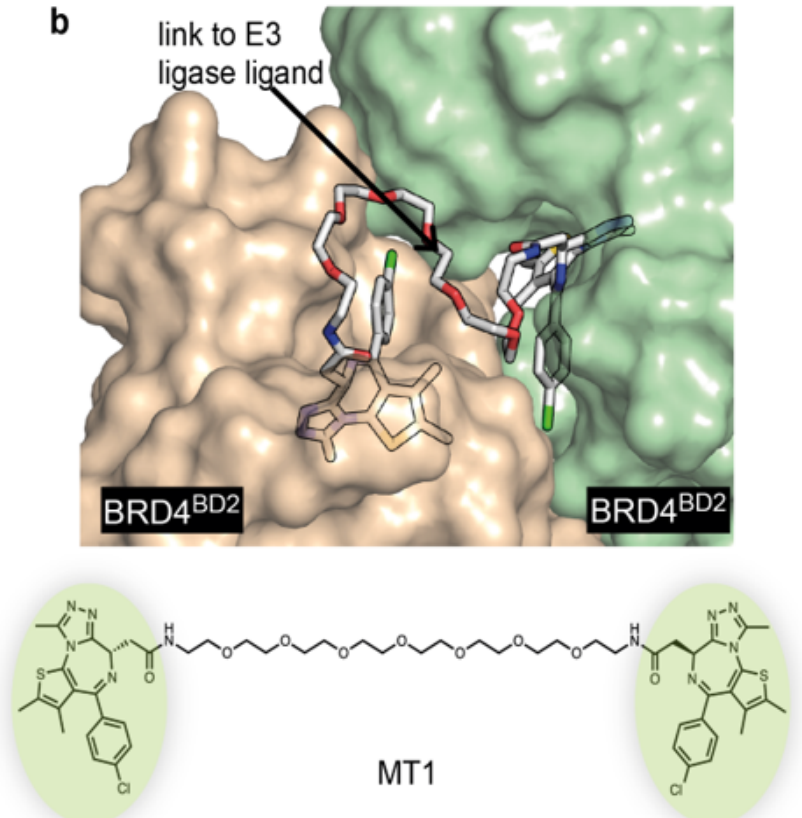

d

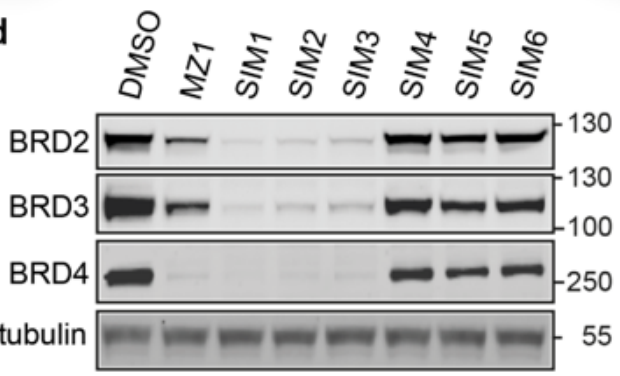

e

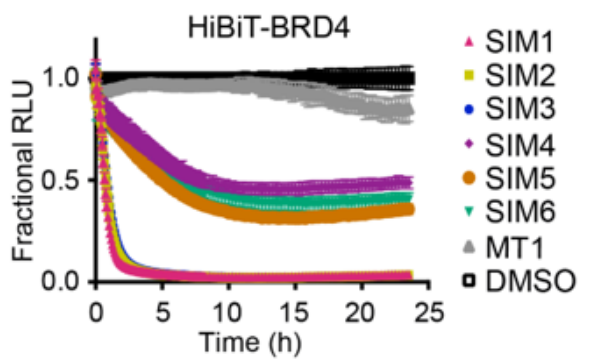

f

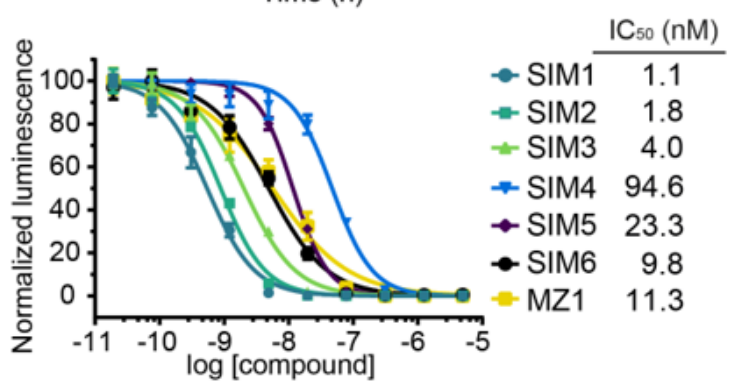

\section{Figure 1}

Structure-inspired design of trivalent PROTACs identifies VHL-based BET degraders. $a, b)$ Inspection of ternary complex crystal structures of VHL:MZ1:BRD4BD2 (a, PDB:5T35) and BRD4BD2:MT1:BRD4BD2 (b, PDB 5JWM) guided the identification of solvent-exposed region for chemical branching of linkers in trivalent PROTAC design. Chemical structures of parent bifunctional molecules MZ1 and MT1 are shown. c) Chemical structures of designed trivalent PROTACs based on VHL and CRBN E3 ligase ligands. d) 
Immunoblot analysis of BRD2, BRD3, BRD4 after treatment of HEK293 cells with $1 \mu \mathrm{M}$ PROTACs or DMSO for $4 \mathrm{~h}$. Full blot can be found in Extended Data Fig. 7. e) Quantitative live-cell degradation kinetics of CRISPR HiBiT-BRD4 HEK293 cells following treatment with $1 \mu \mathrm{M}$ compounds or DMSO in quadruplicates. Luminescence (RLU) was continuously monitored in $5 \mathrm{~min}$ intervals over a $24 \mathrm{~h}$ time period. f) Cell viability of MV4;11 AML cell line following treatment with PROTACs or DMSO for $48 \mathrm{~h}$ in three replicates for each concentration point.
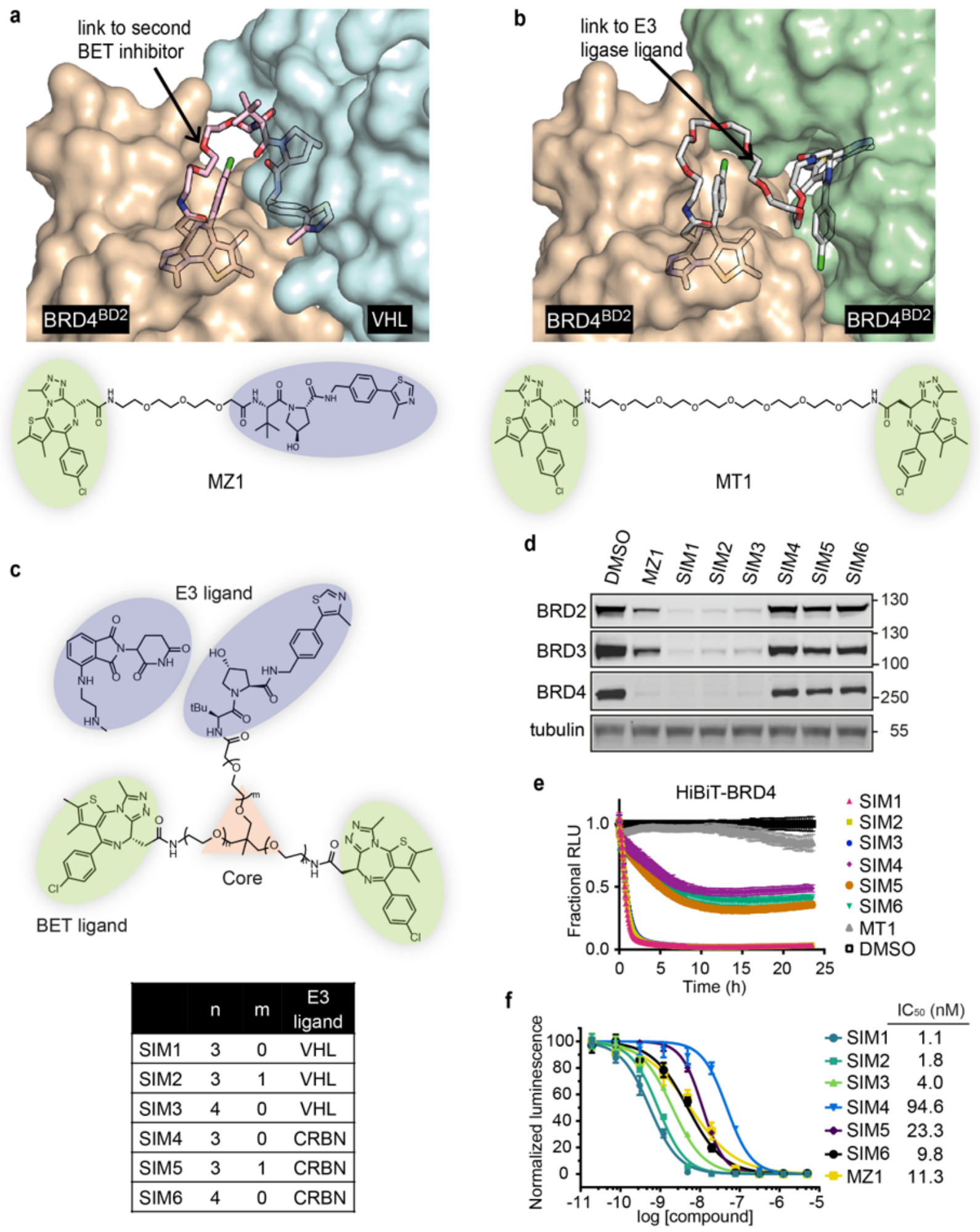

Figure 1

\begin{tabular}{|lccc|} 
& $n$ & $m$ & $\begin{array}{c}\text { E3 } \\
\text { ligand }\end{array}$ \\
\hline SIM1 & 3 & 0 & VHL \\
\hline SIM2 & 3 & 1 & VHL \\
\hline SIM3 & 4 & 0 & VHL \\
\hline SIM4 & 3 & 0 & CRBN \\
\hline SIM5 & 3 & 1 & CRBN \\
\hline SIM6 & 4 & 0 & CRBN \\
\hline
\end{tabular}


Structure-inspired design of trivalent PROTACs identifies VHL-based BET degraders. a,b) Inspection of ternary complex crystal structures of VHL:MZ1:BRD4BD2 (a, PDB:5T35) and BRD4BD2:MT1:BRD4BD2 (b, PDB 5JWM) guided the identification of solvent-exposed region for chemical branching of linkers in trivalent PROTAC design. Chemical structures of parent bifunctional molecules MZ1 and MT1 are shown. c) Chemical structures of designed trivalent PROTACs based on VHL and CRBN E3 ligase ligands. d) Immunoblot analysis of BRD2, BRD3, BRD4 after treatment of HEK293 cells with $1 \mu$ M PROTACs or DMSO for $4 \mathrm{~h}$. Full blot can be found in Extended Data Fig. 7. e) Quantitative live-cell degradation kinetics of CRISPR HiBiT-BRD4 HEK293 cells following treatment with $1 \mu \mathrm{M}$ compounds or DMSO in quadruplicates. Luminescence (RLU) was continuously monitored in 5 min intervals over a $24 \mathrm{~h}$ time period. $\mathrm{f}$ ) Cell viability of MV4;11 AML cell line following treatment with PROTACs or DMSO for $48 \mathrm{~h}$ in three replicates for each concentration point. 
a

\section{DMS}

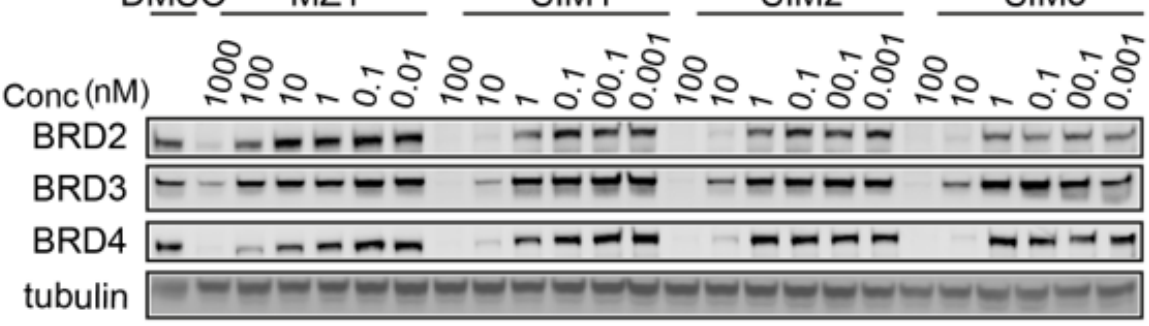

C

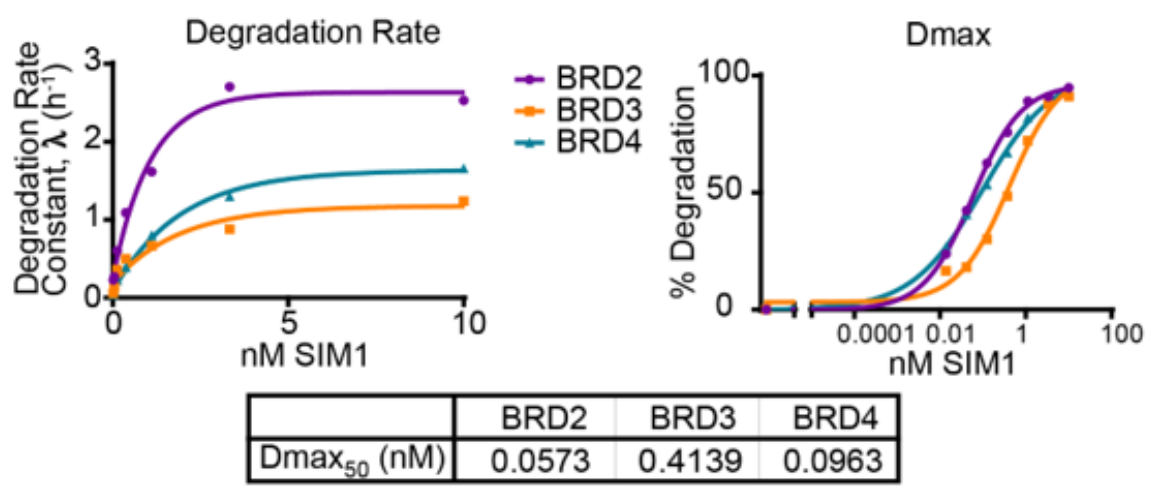

d

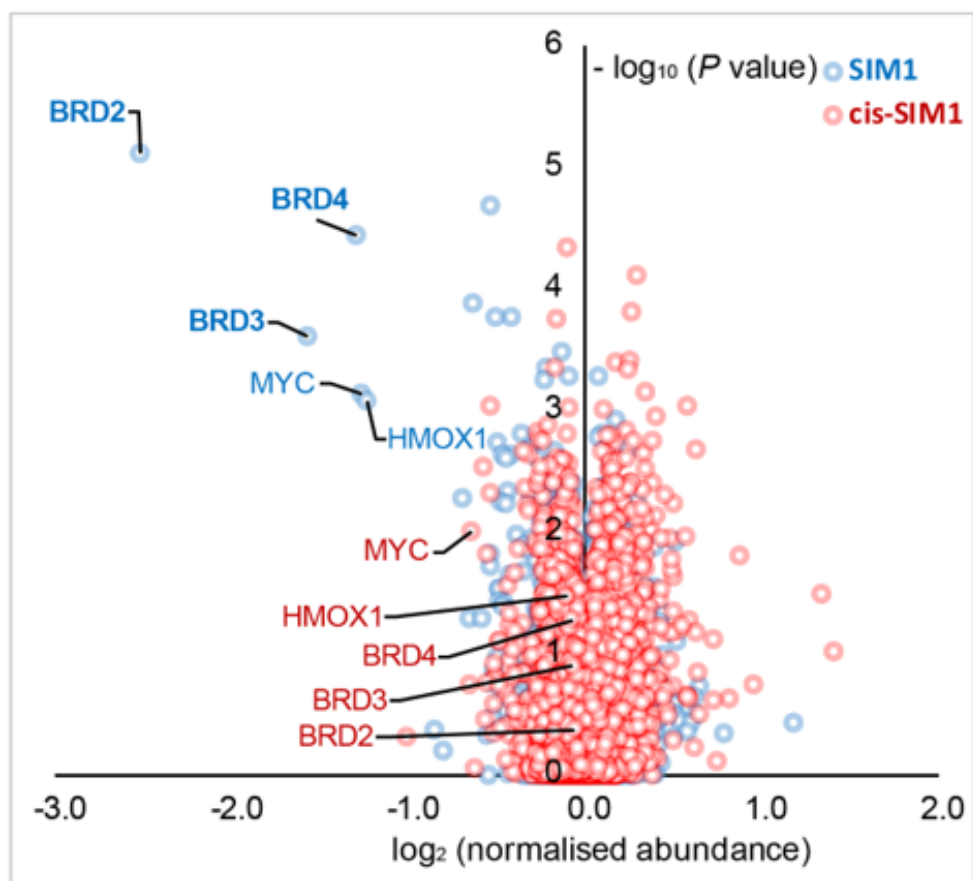

b

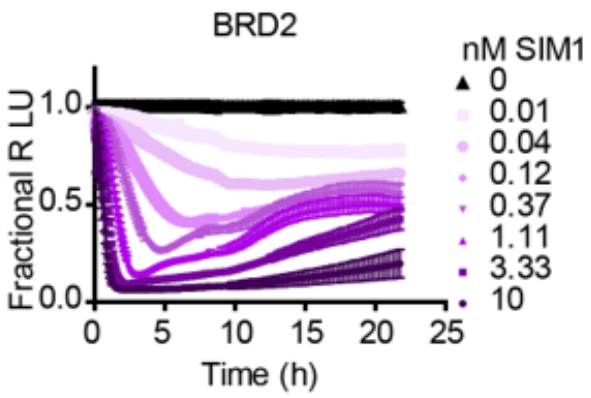

BRD3
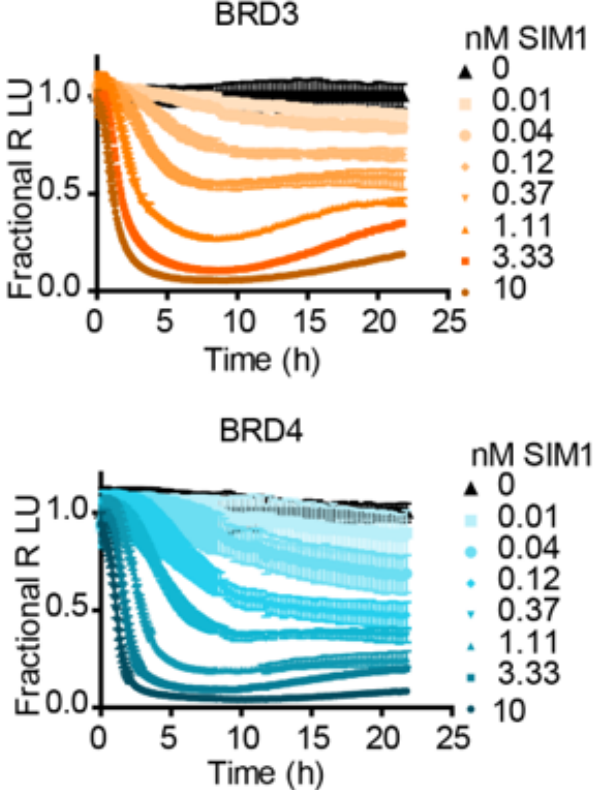

e

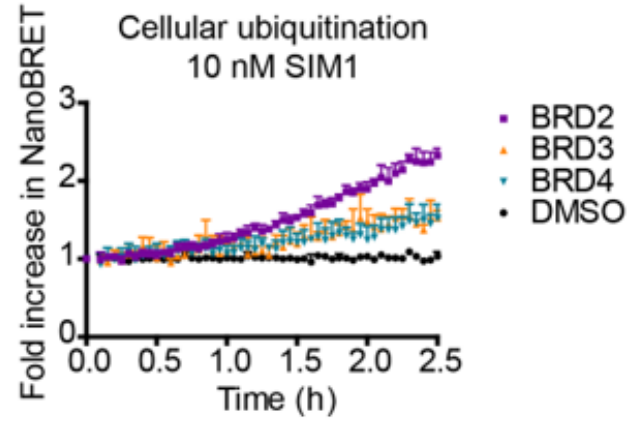

Figure 2

SIM1 is a potent BET PROTAC showing rapid and more profound degradation for BRD2. a) Immunoblot of BRD2, BRD3, BRD4 levels in HEK293 cells treated with serially diluted PROTACs SIM1-SIM3 for $4 \mathrm{~h}$. Quantification of BET protein levels was done relative to DMSO control and plots used to measure DC50 values, as shown in Extended Data Fig. 1d. The blot shown is representative of two independent experiments. Full blots can be found in Extended Data Fig. 7. b) Quantitative live-cell degradation kinetics of CRISPR HiBiT-BRD2, BRD3, and BRD4 HEK293 cells following treatment with serially diluted SIM1 in 
quadruplicates. Luminescence ( $R L U$ ) was continuously monitored over a $24 \mathrm{~h}$ time period. c) Calculation of degradation rate, degradation maximum (Dmax), and Dmax50 values from BRD2, BRD3, and BRD4 kinetic profiles shown in (b). d) Effects of SIM1 (blue) and cis-SIM1 (red) on the proteome of MV4;11 cells treated with compound at $10 \mathrm{nM}$ for $4 \mathrm{~h}$. Data plotted log2 of the normalized fold change in abundance against - $\log 10$ of the $P$ value per protein from three independent experiments. Quantification of representative proteins can be found in Extended Data Fig. 2d. e) NanoBRET ubiquitination kinetics of HiBiT-BET proteins following 10nM SIM1 treatment performed in quadruplicate.

a

DMSO MZ1 $\frac{\mathrm{SIM} 1}{-5} \frac{\mathrm{SIM} 2}{n-S I M 3}$

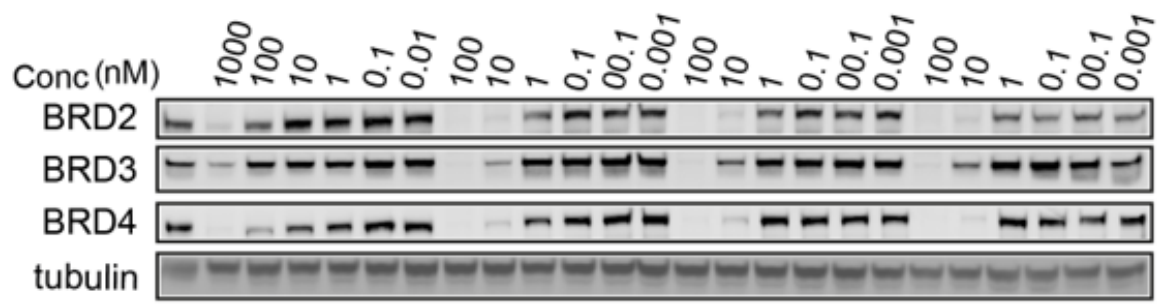

C

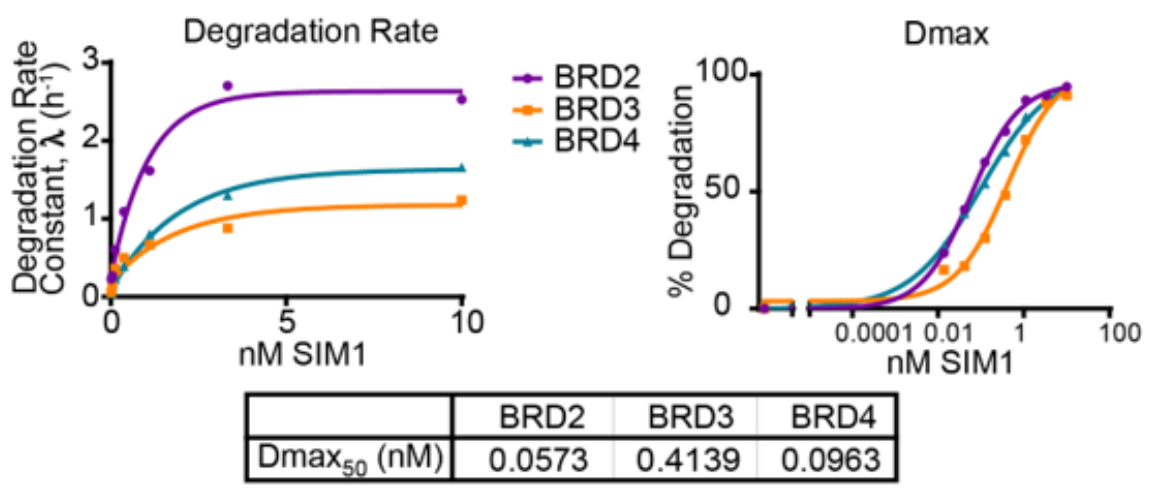

d

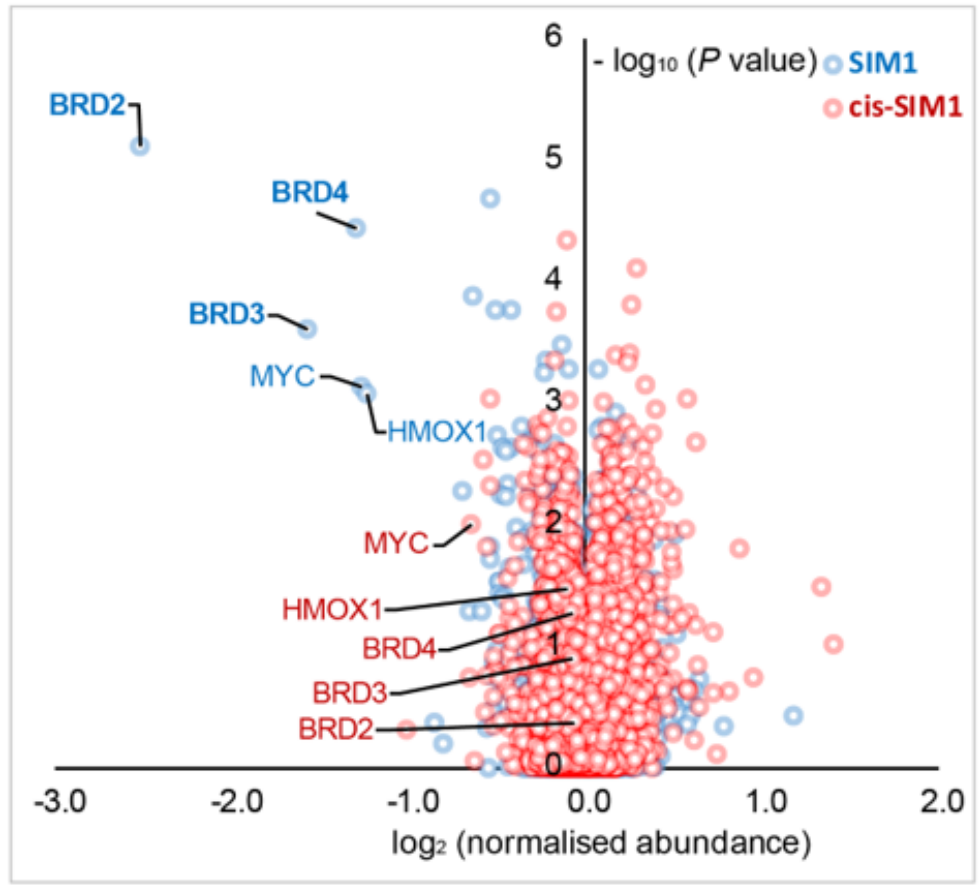

b

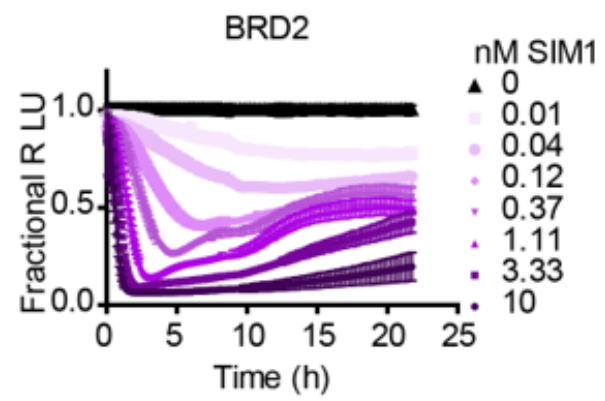

BRD3
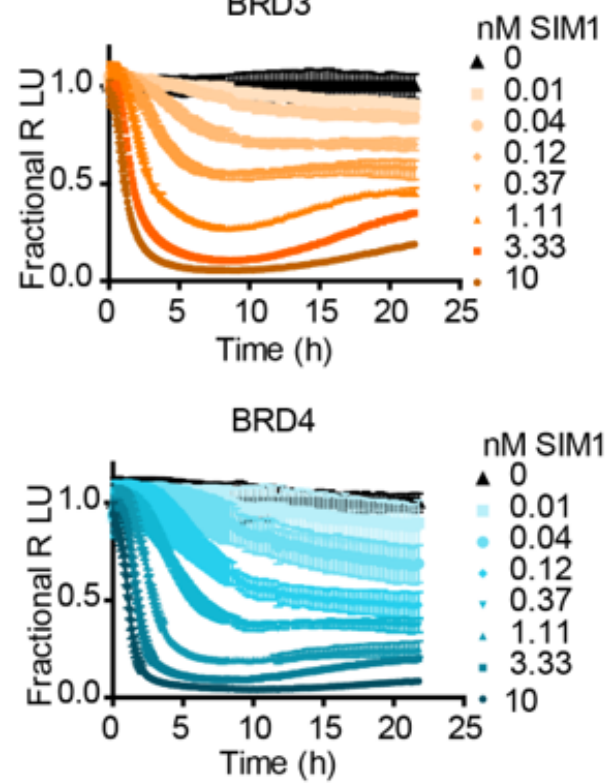

e

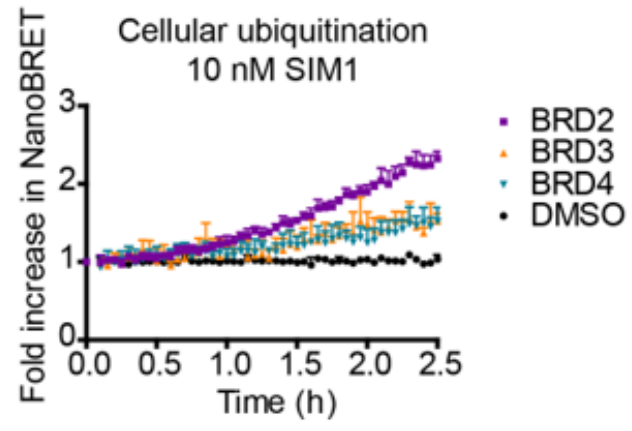

Figure 2 
SIM1 is a potent BET PROTAC showing rapid and more profound degradation for BRD2. a) Immunoblot of BRD2, BRD3, BRD4 levels in HEK293 cells treated with serially diluted PROTACs SIM1-SIM3 for $4 \mathrm{~h}$. Quantification of BET protein levels was done relative to DMSO control and plots used to measure DC50 values, as shown in Extended Data Fig. 1d. The blot shown is representative of two independent experiments. Full blots can be found in Extended Data Fig. 7. b) Quantitative live-cell degradation kinetics of CRISPR HiBiT-BRD2, BRD3, and BRD4 HEK293 cells following treatment with serially diluted SIM1 in quadruplicates. Luminescence (RLU) was continuously monitored over a $24 \mathrm{~h}$ time period. c) Calculation of degradation rate, degradation maximum (Dmax), and Dmax50 values from BRD2, BRD3, and BRD4 kinetic profiles shown in (b). d) Effects of SIM1 (blue) and cis-SIM1 (red) on the proteome of MV4;11 cells treated with compound at $10 \mathrm{nM}$ for $4 \mathrm{~h}$. Data plotted log2 of the normalized fold change in abundance against - $\log 10$ of the $P$ value per protein from three independent experiments. Quantification of representative proteins can be found in Extended Data Fig. 2d. e) NanoBRET ubiquitination kinetics of HiBiT-BET proteins following 10nM SIM1 treatment performed in quadruplicate. 


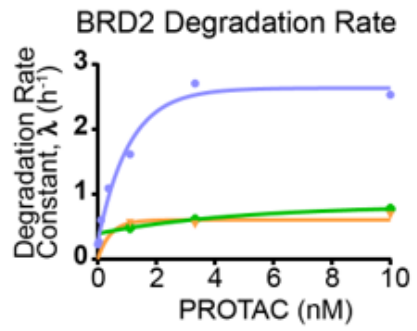

b

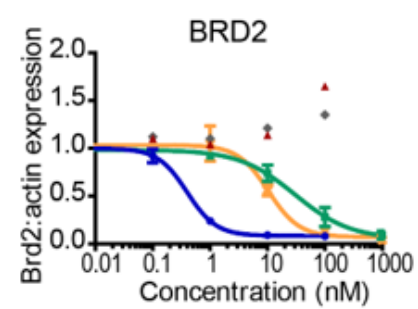

BRD2 Dmax
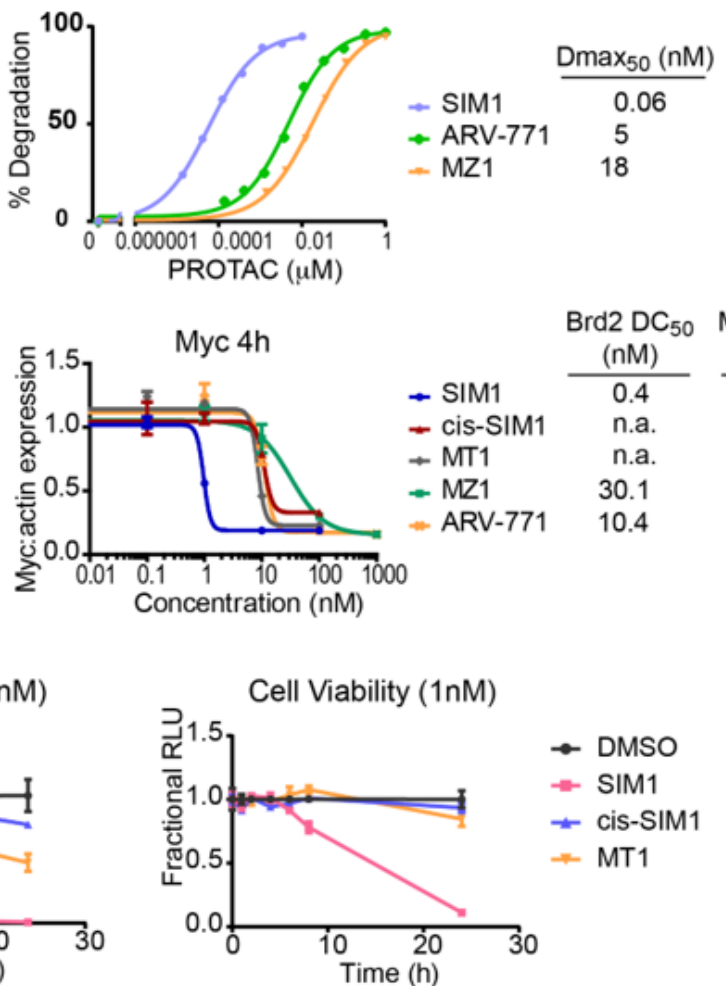

Myc 4h

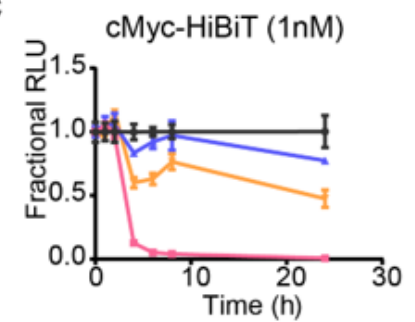

d
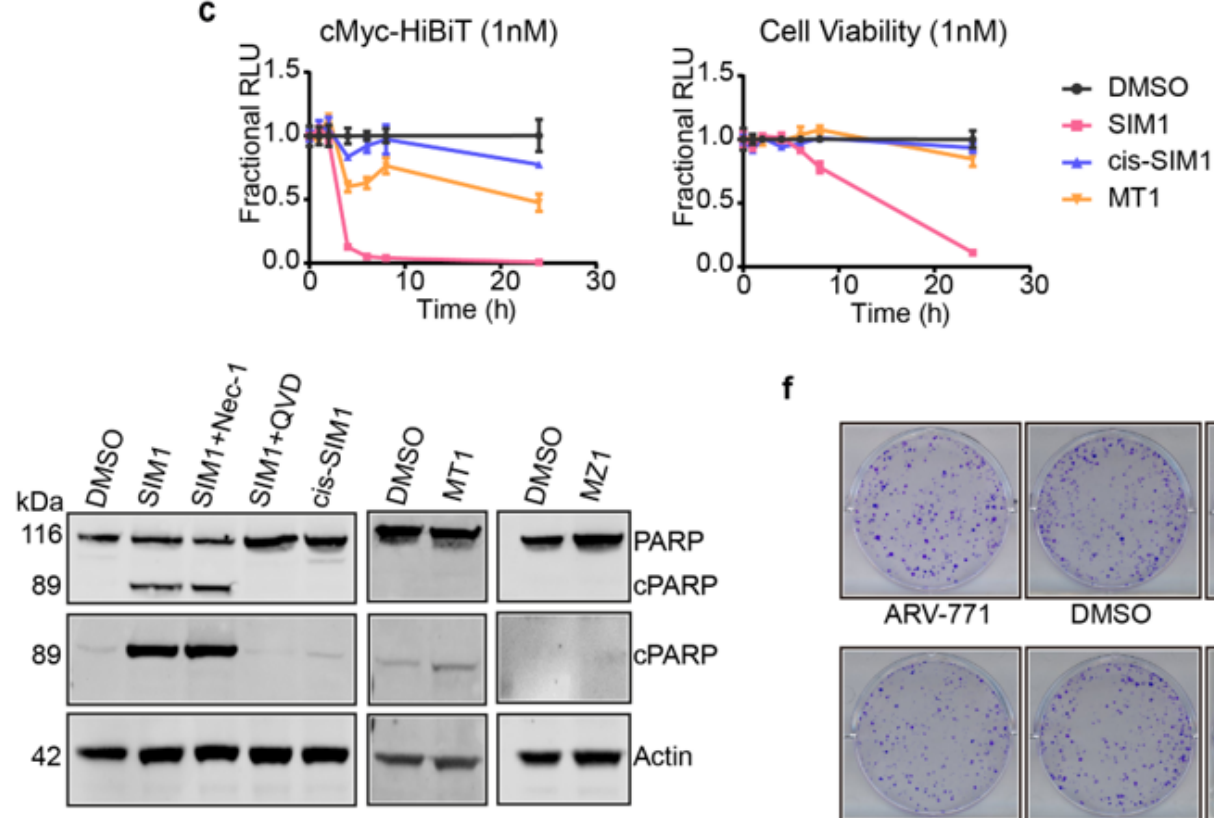

f
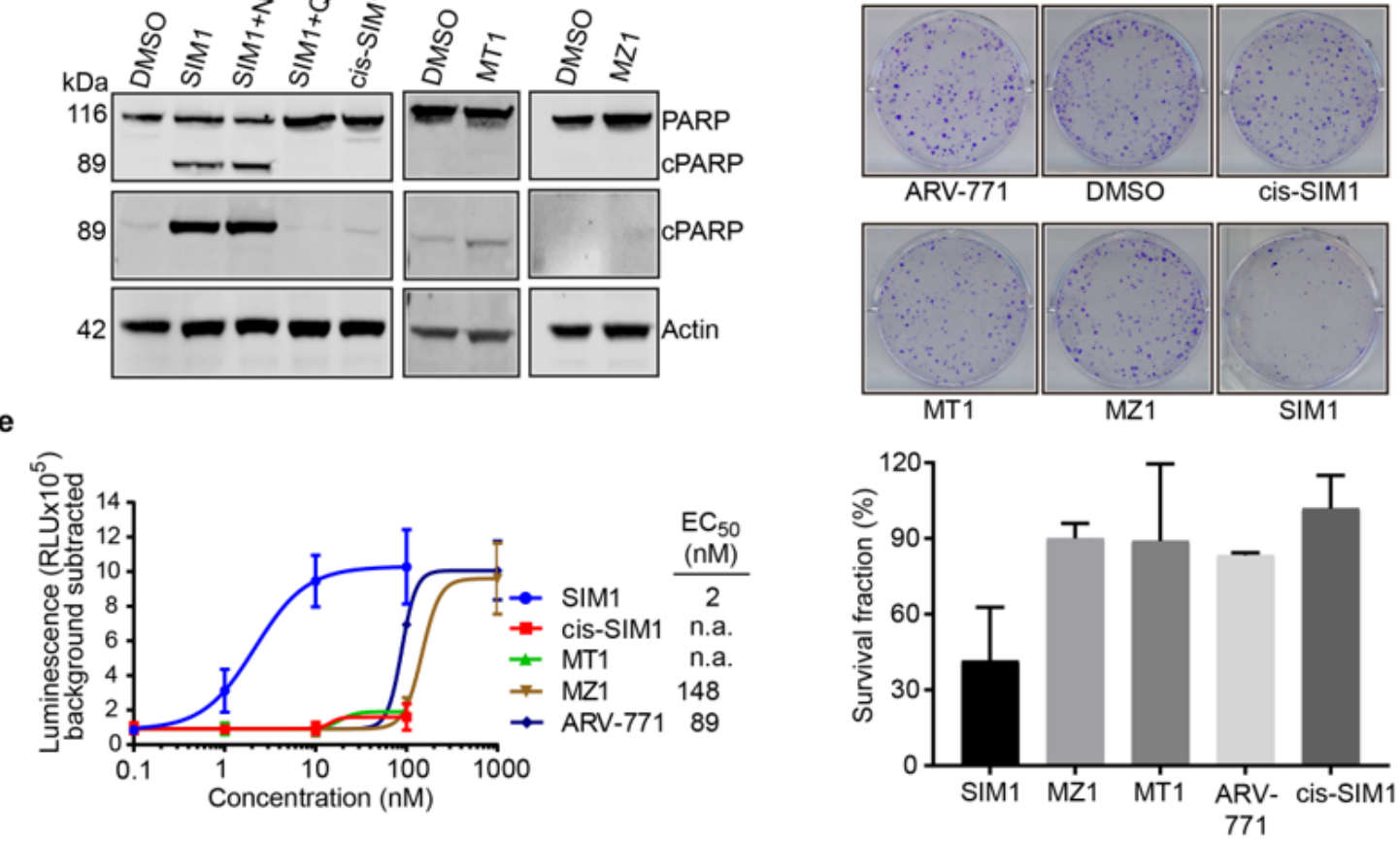

\section{Figure 3}

Trivalent PROTAC SIM1 is functionally more potent than parent bivalent PROTACs and inhibitors. a) Comparison of degradation rate, Dmax, and Dmax50 values calculated from HiBiT-BRD2 kinetic dose response profiles with SIM1 (kinetic profiles show in Fig. 2b), ARV-771 (kinetic profiles shown in Extended Data Fig. 3a), and MZ1 31. b) Quantified expression levels of endogenous BRD2 and Myc in 22Rv1 prostate cancer cell line treated with compounds for $4 \mathrm{~h}$. Curves are a best fit of means from two 
biologically independent experiments, \pm s.e.m. c) Loss in CRISPR cMyc-HiBiT protein levels and correlative cell viability in MV4;11 cells treated with $1 \mathrm{nM}$ concentration of the indicated compounds in quadruplicates. Luminescence and cell viability by CellTiter-Glo were measured at various time points over $24 \mathrm{~h}$. d) Immunoblot of PARP-cleavage in 22Rv1 cells with indicated compounds at 10nM for $24 \mathrm{~h}$ with or without the addition of caspase inhibitor (QVD-OPh, $20 \mu \mathrm{M}$ ) or necroptosis inhibitor (Necrostatin-1, $20 \mu \mathrm{M})$. Blots for $48 \mathrm{~h}$ treatments and $1 \mu \mathrm{M} \mathrm{MZ1}$ and MT1 treatments are in Extended Data Fig. 5. Full blots can be found in Extended Data Fig. 8. e) Caspase-Glo 3/7 assays treated with compounds or DMSO for $24 \mathrm{~h}$ in 22Rv1 cells. Curves are a best fit of means from three biologically independent experiments, \pm s.e.m. f) Survival of $22 R v 1$ cells in clonogenic assay. Cells were treated with $10 \mathrm{nM}$ compounds for $24 \mathrm{~h}$. Five hundred cells were re-plated and allowed to grow at $37^{\circ} \mathrm{C}$ for 20 days before scanning. Survival fraction was determined by dividing plating efficiency of treated cells by plating efficiency of untreated cells. Error bars indicate the mean values \pm S.D. from duplicates. 


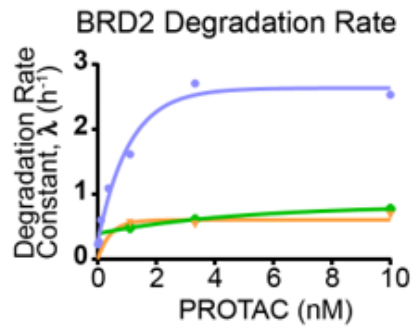

b

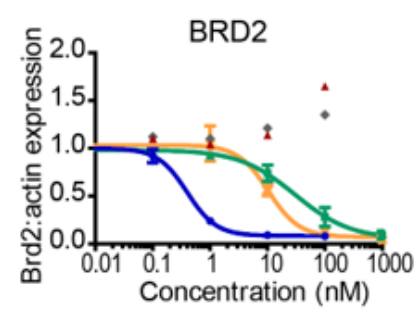

BRD2 Dmax
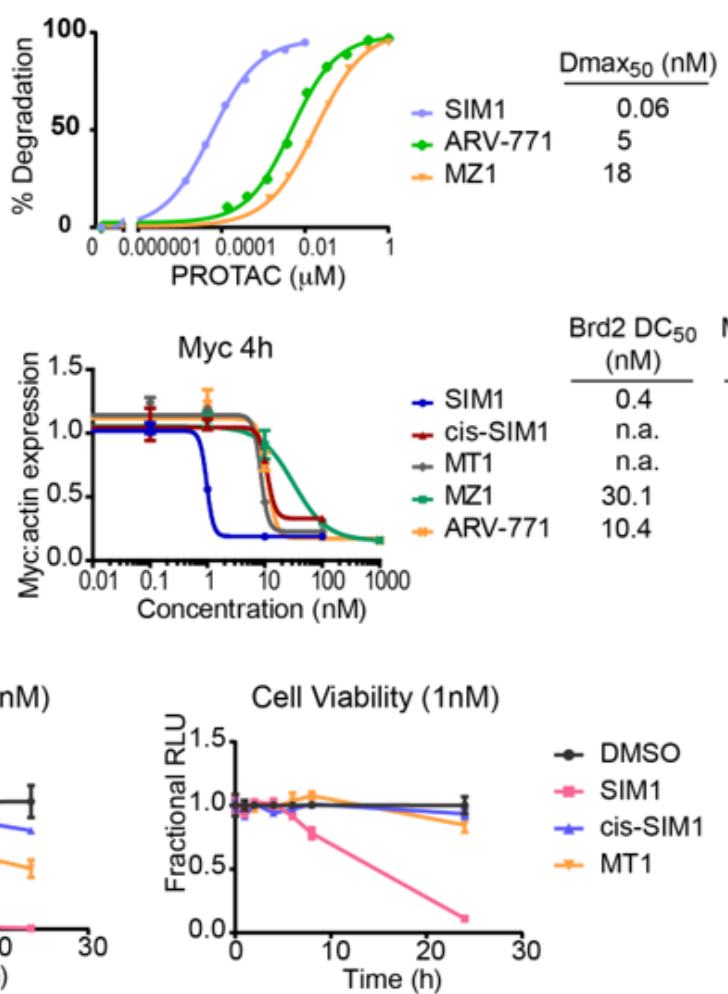

Myc 4h

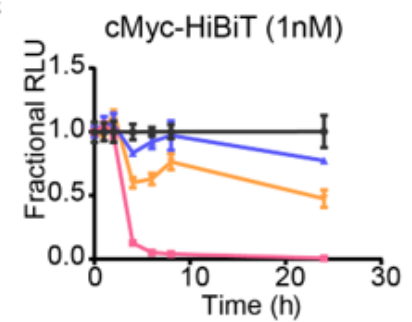

d
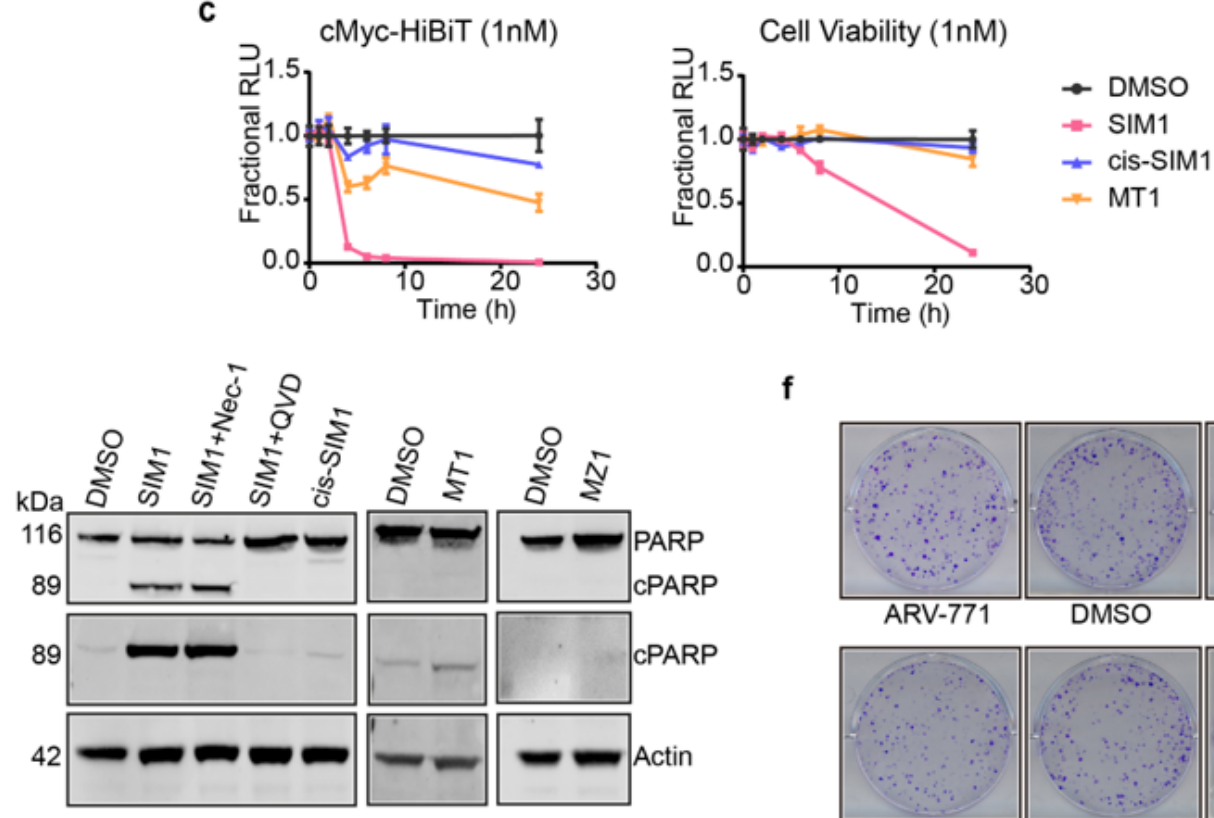

f
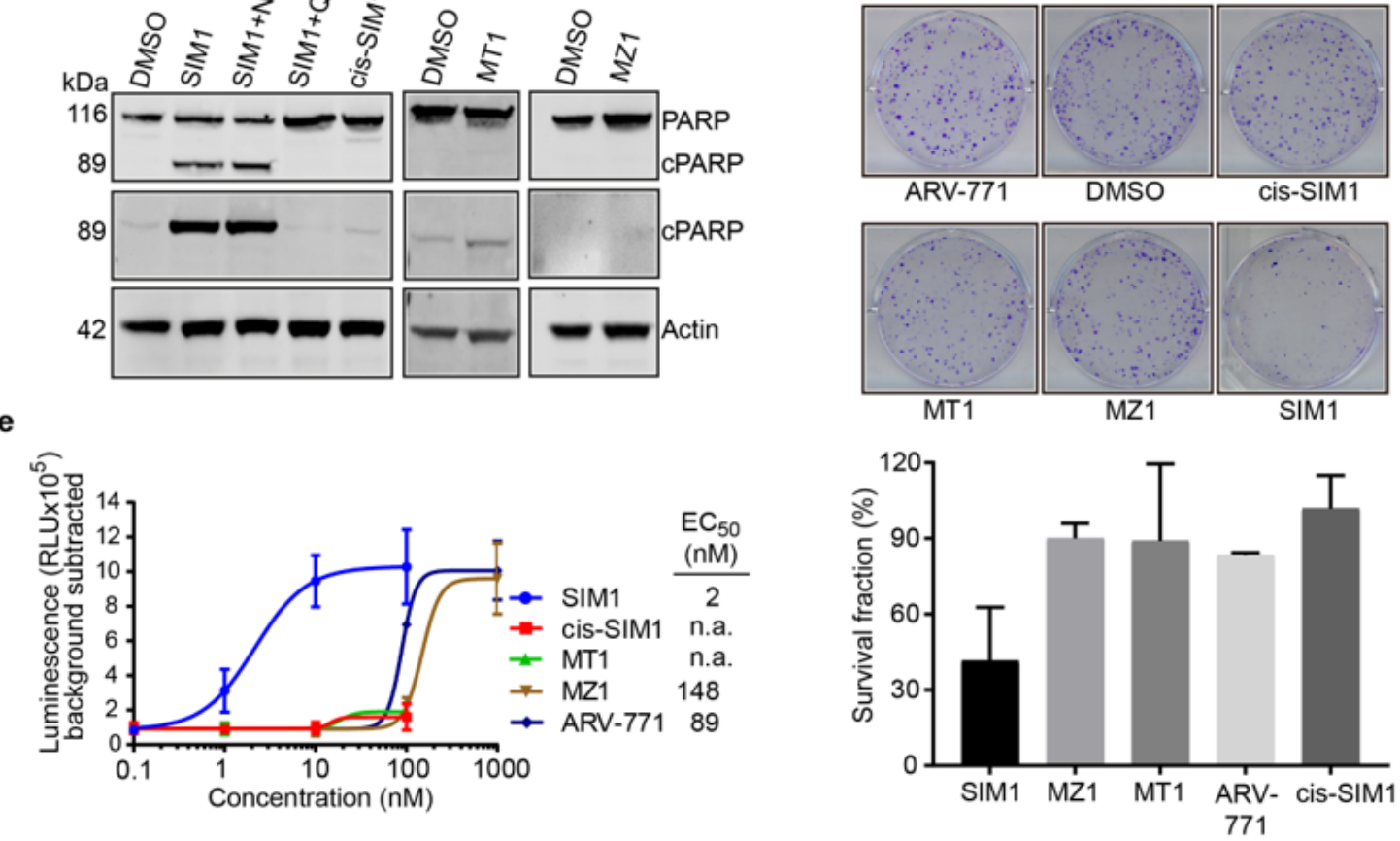

\section{Figure 3}

Trivalent PROTAC SIM1 is functionally more potent than parent bivalent PROTACs and inhibitors. a) Comparison of degradation rate, Dmax, and Dmax50 values calculated from HiBiT-BRD2 kinetic dose response profiles with SIM1 (kinetic profiles show in Fig. 2b), ARV-771 (kinetic profiles shown in Extended Data Fig. 3a), and MZ1 31. b) Quantified expression levels of endogenous BRD2 and Myc in 22Rv1 prostate cancer cell line treated with compounds for $4 \mathrm{~h}$. Curves are a best fit of means from two 
biologically independent experiments, \pm s.e.m. c) Loss in CRISPR cMyc-HiBiT protein levels and correlative cell viability in MV4;11 cells treated with $1 \mathrm{nM}$ concentration of the indicated compounds in quadruplicates. Luminescence and cell viability by CellTiter-Glo were measured at various time points over $24 \mathrm{~h}$. d) Immunoblot of PARP-cleavage in 22Rv1 cells with indicated compounds at 10nM for $24 \mathrm{~h}$ with or without the addition of caspase inhibitor (QVD-OPh, $20 \mu \mathrm{M}$ ) or necroptosis inhibitor (Necrostatin-1, $20 \mu \mathrm{M})$. Blots for $48 \mathrm{~h}$ treatments and $1 \mu \mathrm{M} \mathrm{MZ1}$ and MT1 treatments are in Extended Data Fig. 5. Full blots can be found in Extended Data Fig. 8. e) Caspase-Glo 3/7 assays treated with compounds or DMSO for $24 \mathrm{~h}$ in 22Rv1 cells. Curves are a best fit of means from three biologically independent experiments, \pm s.e.m. f) Survival of $22 R v 1$ cells in clonogenic assay. Cells were treated with $10 \mathrm{nM}$ compounds for $24 \mathrm{~h}$. Five hundred cells were re-plated and allowed to grow at $37^{\circ} \mathrm{C}$ for 20 days before scanning. Survival fraction was determined by dividing plating efficiency of treated cells by plating efficiency of untreated cells. Error bars indicate the mean values \pm S.D. from duplicates. 

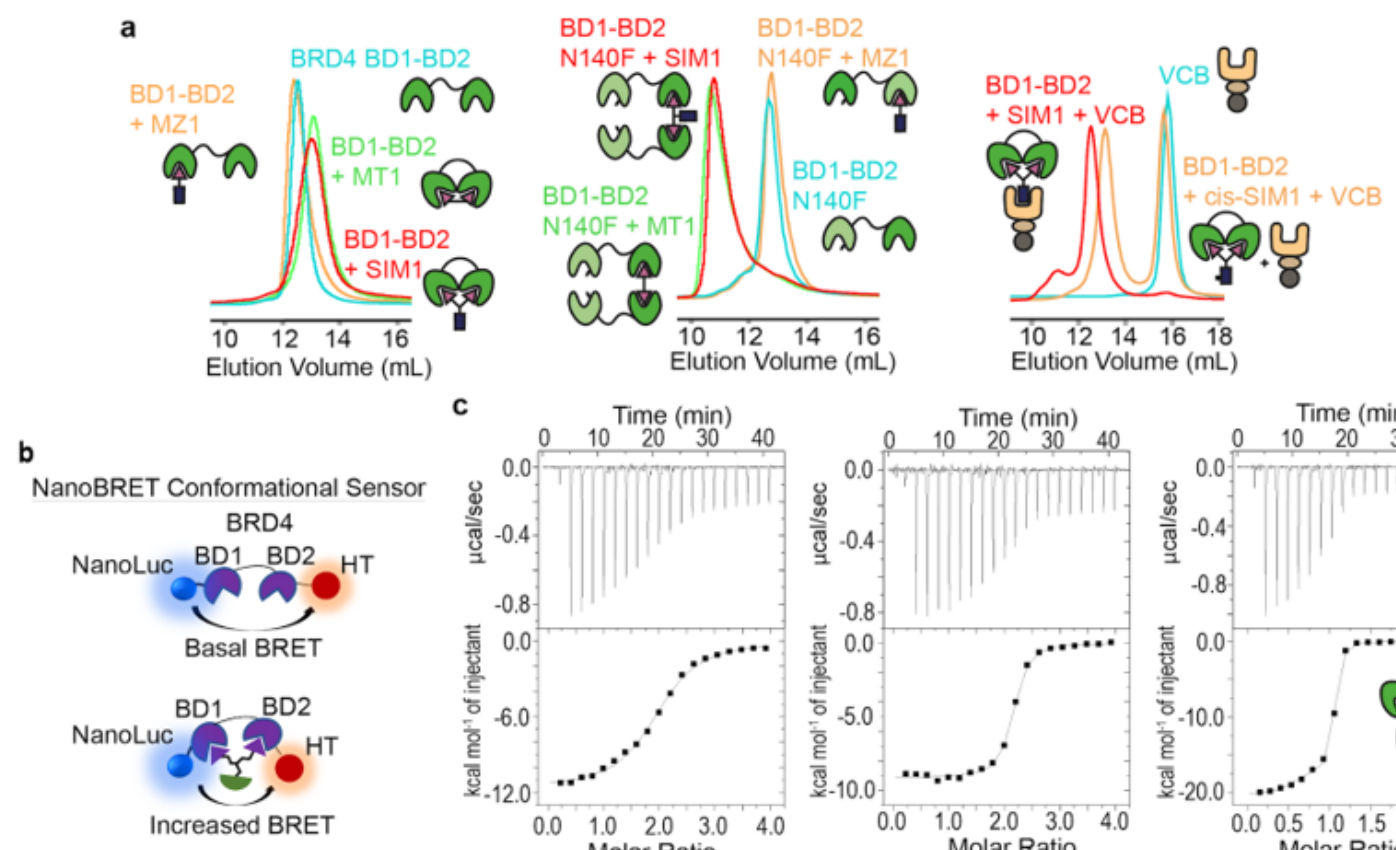

c
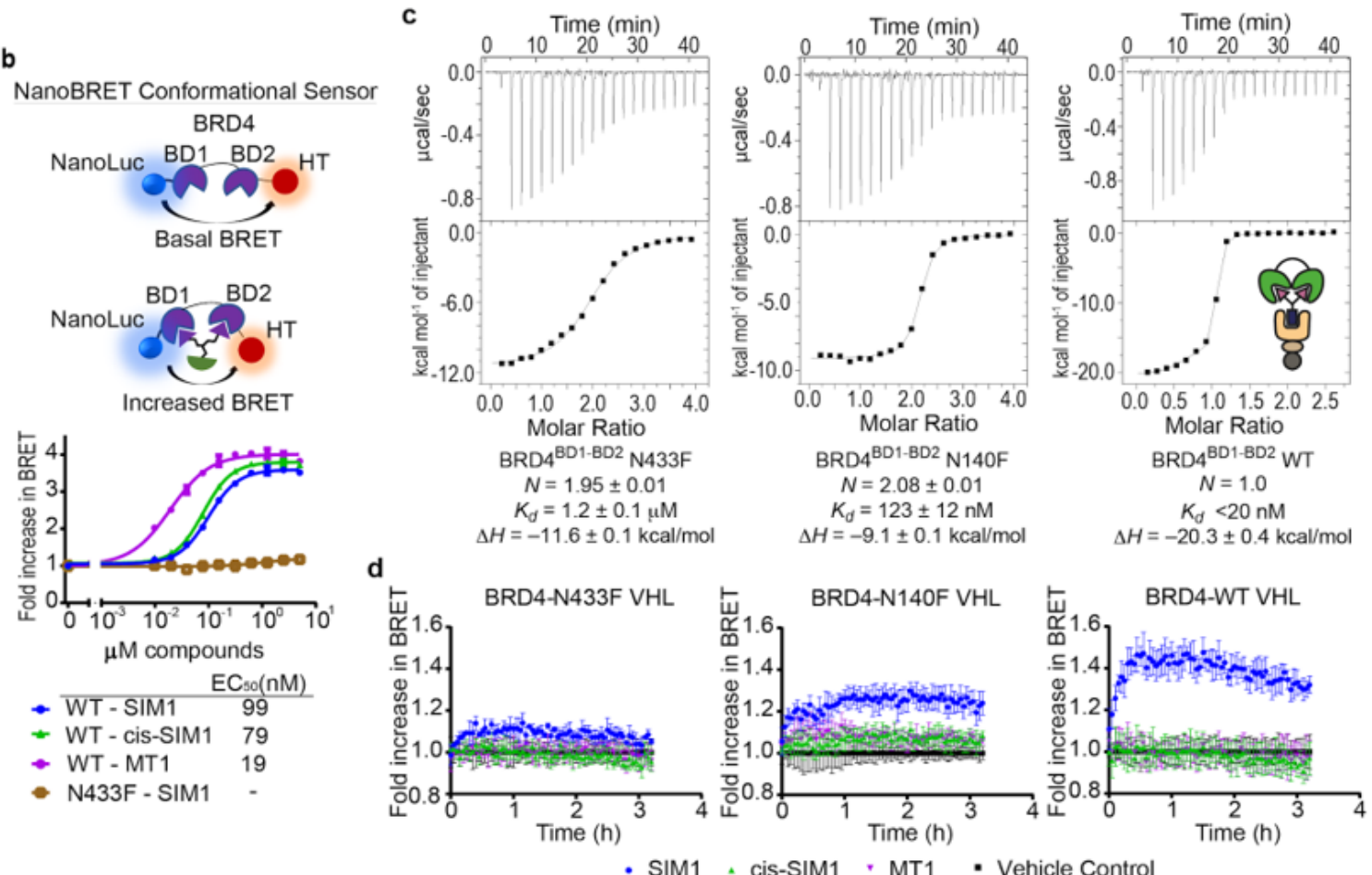

e

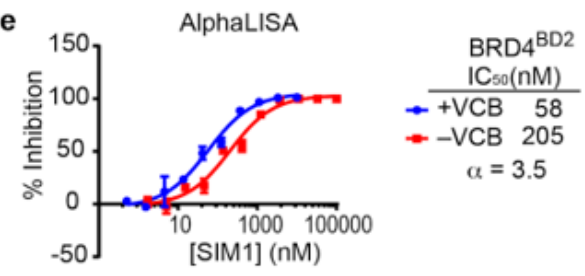

g

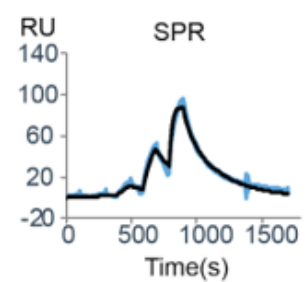

SIM1:BRD4 $4^{\text {BD1-BO2 }}$ vs immobilized VCB

$\mathrm{K}_{\mathrm{d}}: 53 \pm 4 \mathrm{nM}$

$t_{1 / 2}: 119 \pm 21 s$
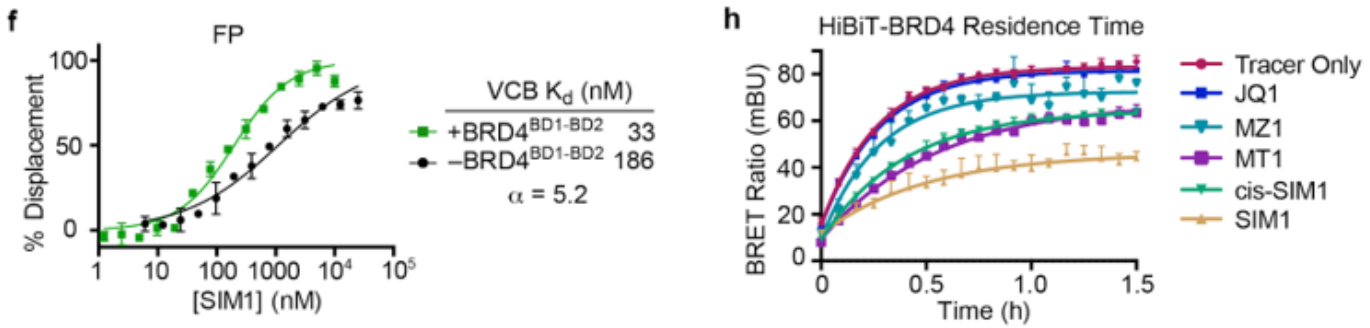

\section{Figure 4}

SIM1 intramolecularly engages BD1-BD2 tandem domain to form stable and cooperative 1:1:1 VHL:SIM1:BRD4 complexes with prolonged cellular residence time. a) Size exclusion chromatography of complex formation after incubation of SIM1 (red), MZ1 or cis-SIM1 (orange), MT1 (green) or DMSO (cyan) with BD1-BD2 tandem domain from BRD4 (left panel: wild type, middle panel: N140F mutant, right panel: wild type with VCB protein). Intensity of peaks is absorbance at $280 \mathrm{~nm}$. b) NanoBRET 
conformational biosensor assay consisting of either the BD1-BD2 tandem domain of BRD4 wild-type (WT) or containing the BD2 N433F mutation flanked by NanoLuc donor and HaloTag acceptor fusion tags. HEK293 cells were transiently transfected with either the WT or N433F mutant biosensor and treated with a serial dilution of SIM1, cis-SIM1, or MT1 compounds in quadruplicates. NanoBRET was measured to determine a change in tag proximity indicative of a conformational change For treatments which showed a conformational change, EC50 values were calculated and are shown. C) ITC titrations of BRD4 BD1-BD2 tandem proteins (loaded in the syringe, N-to-F mutants at $300 \mu \mathrm{M}$, WT $200 \mu \mathrm{M}$ ) into a 1:1 mixture of SIM1 $(16 \mu \mathrm{M})$ and VCB $(32 \mu \mathrm{M})$ pre-incubated into the sample cell. d) NanoBRET kinetic ternary complex formation in HEK293 cells transiently expressing HaloTag-VHL paired with either fulllength BRD4 WT, N140F or N433F mutants treated with SIM1, cis-SIM1, MT1 or DMSO control in quadruplicates. NanoBRET was continuously monitored for $2 \mathrm{~h}$ after compound addition and showed differential levels of ternary complex formation for each BRD4 variant. e) AlphaLISA titrations of SIM1 in duplicates against biotin-JQ1:BRD4BD2 in the absence (red) or presence (blue) of VCB protein. f) Fitted curves from fluorescence polarization competition assays measuring displacement of a FAM-labelled HIF-1 a peptide from VCB by SIM1 titrated in triplicates, in the presence or absence of BRD4 tandem BD1BD2. g) SPR sensogram to monitor in real-time the interaction of binary complex SIM1:BRD4-BD1-BD2tandem with immobilized biotin-VCB protein. Sensorgram shown is for a ternary single-cycle kinetic (SCK) experiment as representative to three independent experiments, to measure dissociation constant $\mathrm{Kd}$ and dissociative half-life t1/2 of the ternary complex. h) Live cell compound residence time experiments with BRD4 as measured by NanoBRET target engagement. CRISPR HiBiT-BRD4 cells were incubated with the indicated compounds in quadruplicate followed by addition of a competitive fluorescently-labeled BET inhibitor. NanoBRET was measured kinetically in live cells. 

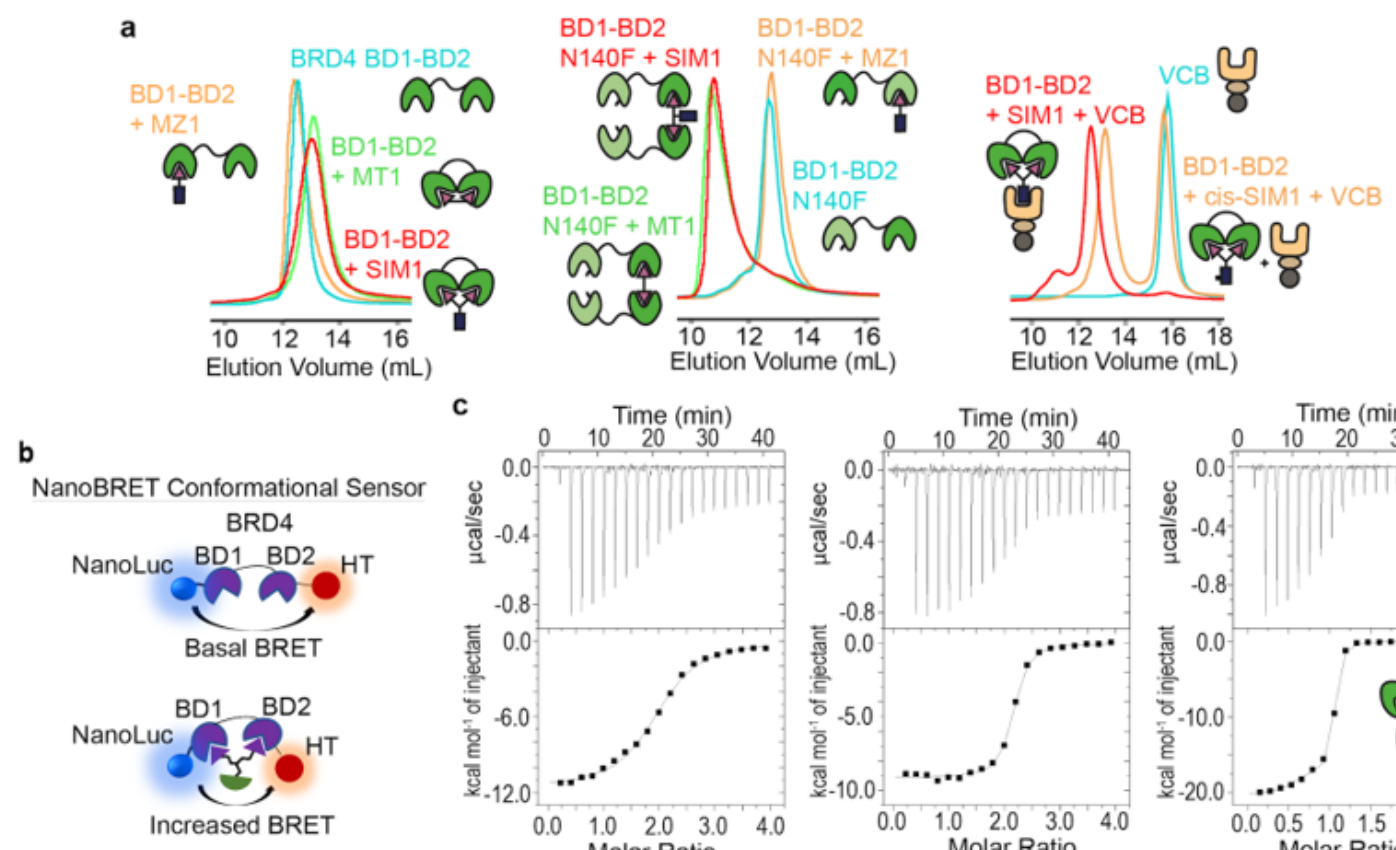

c
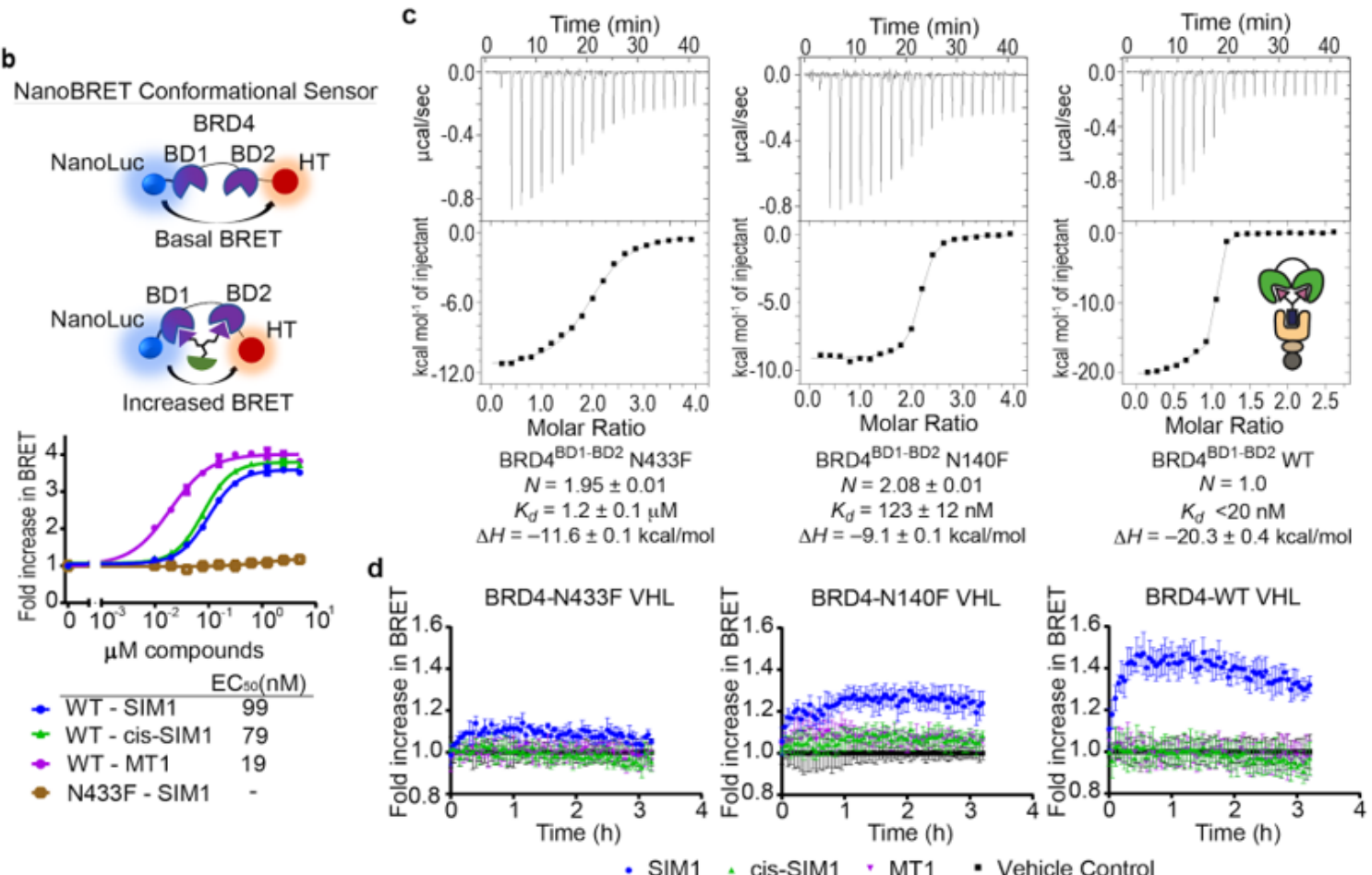

e

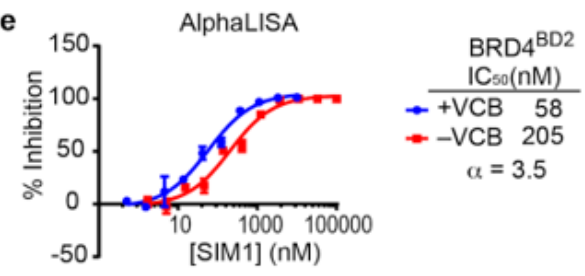

g

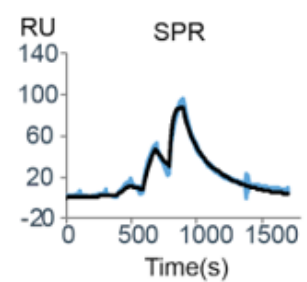

SIM1:BRD4 $4^{\text {BD1-BO2 }}$ vs immobilized VCB

$\mathrm{K}_{\mathrm{d}}: 53 \pm 4 \mathrm{nM}$

$t_{1 / 2}: 119 \pm 21 s$
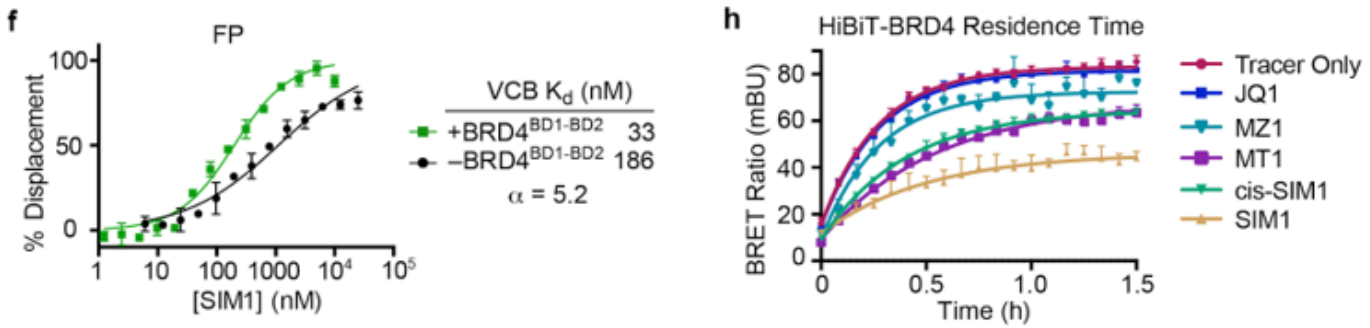

\section{Figure 4}

SIM1 intramolecularly engages BD1-BD2 tandem domain to form stable and cooperative 1:1:1 VHL:SIM1:BRD4 complexes with prolonged cellular residence time. a) Size exclusion chromatography of complex formation after incubation of SIM1 (red), MZ1 or cis-SIM1 (orange), MT1 (green) or DMSO (cyan) with BD1-BD2 tandem domain from BRD4 (left panel: wild type, middle panel: N140F mutant, right panel: wild type with VCB protein). Intensity of peaks is absorbance at $280 \mathrm{~nm}$. b) NanoBRET 
conformational biosensor assay consisting of either the BD1-BD2 tandem domain of BRD4 wild-type (WT) or containing the BD2 N433F mutation flanked by NanoLuc donor and HaloTag acceptor fusion tags. HEK293 cells were transiently transfected with either the WT or N433F mutant biosensor and treated with a serial dilution of SIM1, cis-SIM1, or MT1 compounds in quadruplicates. NanoBRET was measured to determine a change in tag proximity indicative of a conformational change For treatments which showed a conformational change, EC50 values were calculated and are shown. C) ITC titrations of BRD4 BD1-BD2 tandem proteins (loaded in the syringe, N-to-F mutants at $300 \mu \mathrm{M}$, WT $200 \mu \mathrm{M}$ ) into a 1:1 mixture of SIM1 $(16 \mu \mathrm{M})$ and VCB $(32 \mu \mathrm{M})$ pre-incubated into the sample cell. d) NanoBRET kinetic ternary complex formation in HEK293 cells transiently expressing HaloTag-VHL paired with either fulllength BRD4 WT, N140F or N433F mutants treated with SIM1, cis-SIM1, MT1 or DMSO control in quadruplicates. NanoBRET was continuously monitored for $2 \mathrm{~h}$ after compound addition and showed differential levels of ternary complex formation for each BRD4 variant. e) AlphaLISA titrations of SIM1 in duplicates against biotin-JQ1:BRD4BD2 in the absence (red) or presence (blue) of VCB protein. f) Fitted curves from fluorescence polarization competition assays measuring displacement of a FAM-labelled HIF-1 a peptide from VCB by SIM1 titrated in triplicates, in the presence or absence of BRD4 tandem BD1BD2. g) SPR sensogram to monitor in real-time the interaction of binary complex SIM1:BRD4-BD1-BD2tandem with immobilized biotin-VCB protein. Sensorgram shown is for a ternary single-cycle kinetic (SCK) experiment as representative to three independent experiments, to measure dissociation constant $\mathrm{Kd}$ and dissociative half-life t1/2 of the ternary complex. h) Live cell compound residence time experiments with BRD4 as measured by NanoBRET target engagement. CRISPR HiBiT-BRD4 cells were incubated with the indicated compounds in quadruplicate followed by addition of a competitive fluorescently-labeled BET inhibitor. NanoBRET was measured kinetically in live cells. 


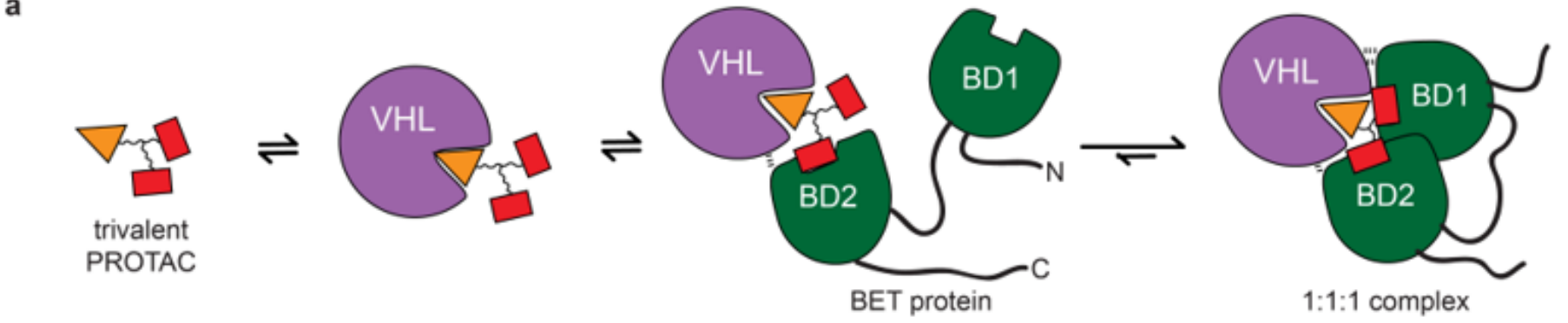

E3 ligase engagement preferential BD2 recruitment

cis-intramolecular engagement with conformational change avidity- and cooperativity- enhanced ternary complex

b

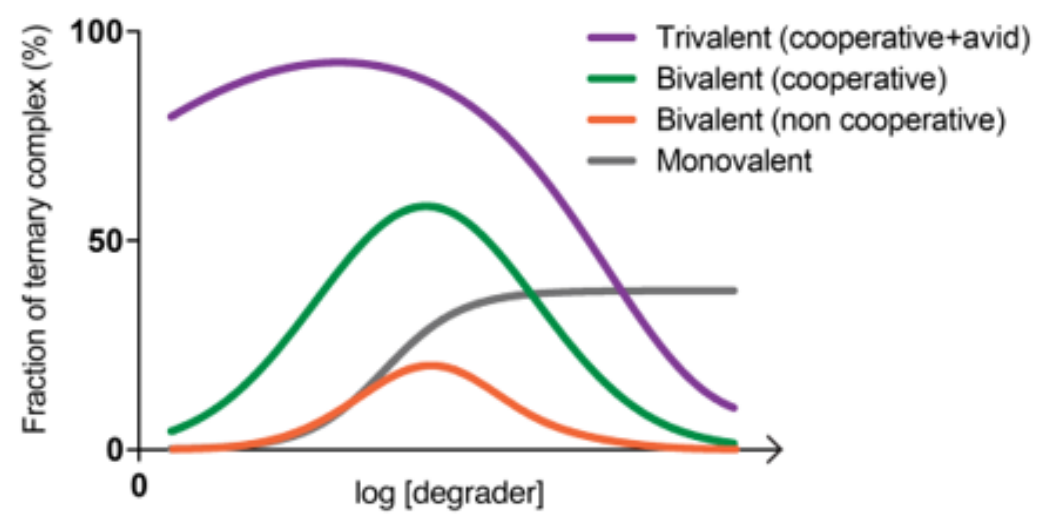

C

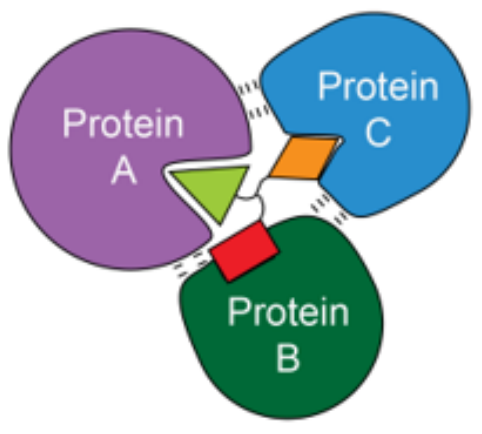

modular trifunctional agent for proximity-induced modalities

\section{Figure 5}

Models of trivalent ternary complex formations and advantages over monovalent and bivalent compounds. a) Proposed mechanism for the formation of a 1:1:1 ternary complex between trivalent PROTAC, VHL and BET protein. Preferential initial binding of the PROTAC to BD2 of BRD4 is followed by conformational change and bidentate binding to BD1. Avidity and cooperativity contribute to formation of a highly stable ternary complex with enhanced residence time at extraordinarily low concentrations of SIM1. b) Shown are different types of degrader-induced ternary complexes e.g. between a target protein and an E3 ligase, depicted at their varying extents as a function of degrader concentration for monovalent. A trivalent complex combining avidity with cooperativity shows the highest and most sustained levels of ternary complex formation, with a minimized hook effect. A cooperative bivalent PROTAC complex is next, followed by a non-cooperative bivalent complex. Lastly, the ternary complex induced by molecular glue compounds is shown, which reaches a plateau and unlike PROTACs are not predicted to experience the competitive hook effect at higher concentrations. c) A general model for trifunctional compound-induced ternary complex utilizing a compound with three different warheads (or ligands) to recruit together three distinct protein. 


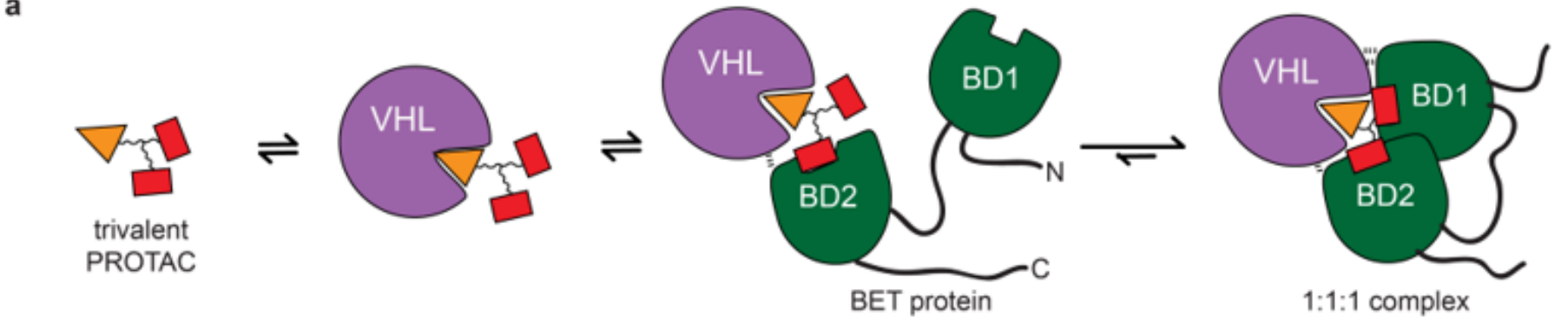

E3 ligase engagement preferential BD2 recruitment

cis-intramolecular engagement with conformational change avidity- and cooperativity- enhanced ternary complex

b

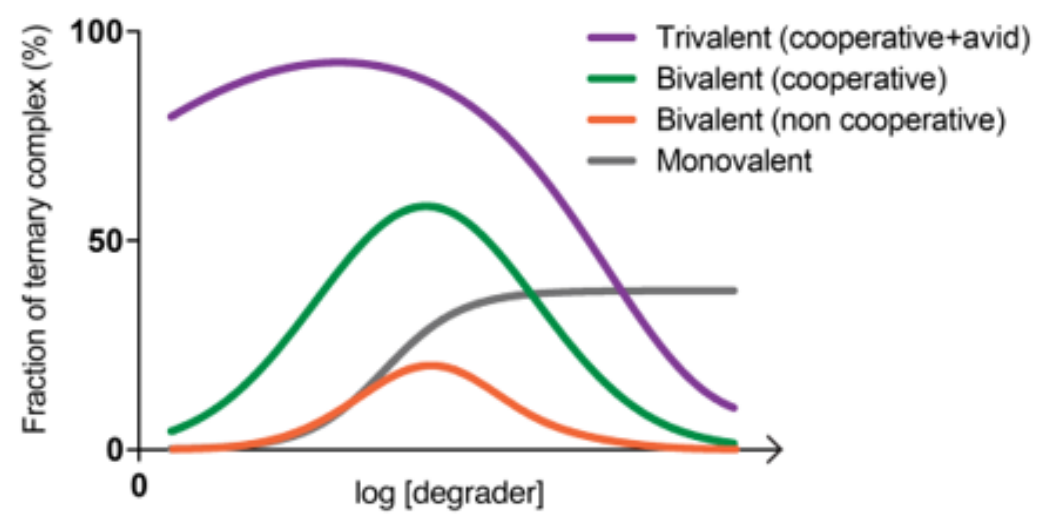

C

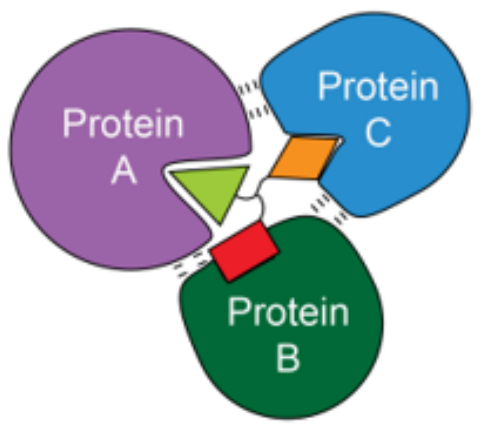

modular trifunctional agent for proximity-induced modalities

\section{Figure 5}

Models of trivalent ternary complex formations and advantages over monovalent and bivalent compounds. a) Proposed mechanism for the formation of a 1:1:1 ternary complex between trivalent PROTAC, VHL and BET protein. Preferential initial binding of the PROTAC to BD2 of BRD4 is followed by conformational change and bidentate binding to BD1. Avidity and cooperativity contribute to formation of a highly stable ternary complex with enhanced residence time at extraordinarily low concentrations of SIM1. b) Shown are different types of degrader-induced ternary complexes e.g. between a target protein and an E3 ligase, depicted at their varying extents as a function of degrader concentration for monovalent. A trivalent complex combining avidity with cooperativity shows the highest and most sustained levels of ternary complex formation, with a minimized hook effect. A cooperative bivalent PROTAC complex is next, followed by a non-cooperative bivalent complex. Lastly, the ternary complex induced by molecular glue compounds is shown, which reaches a plateau and unlike PROTACs are not predicted to experience the competitive hook effect at higher concentrations. c) A general model for trifunctional compound-induced ternary complex utilizing a compound with three different warheads (or ligands) to recruit together three distinct protein. 

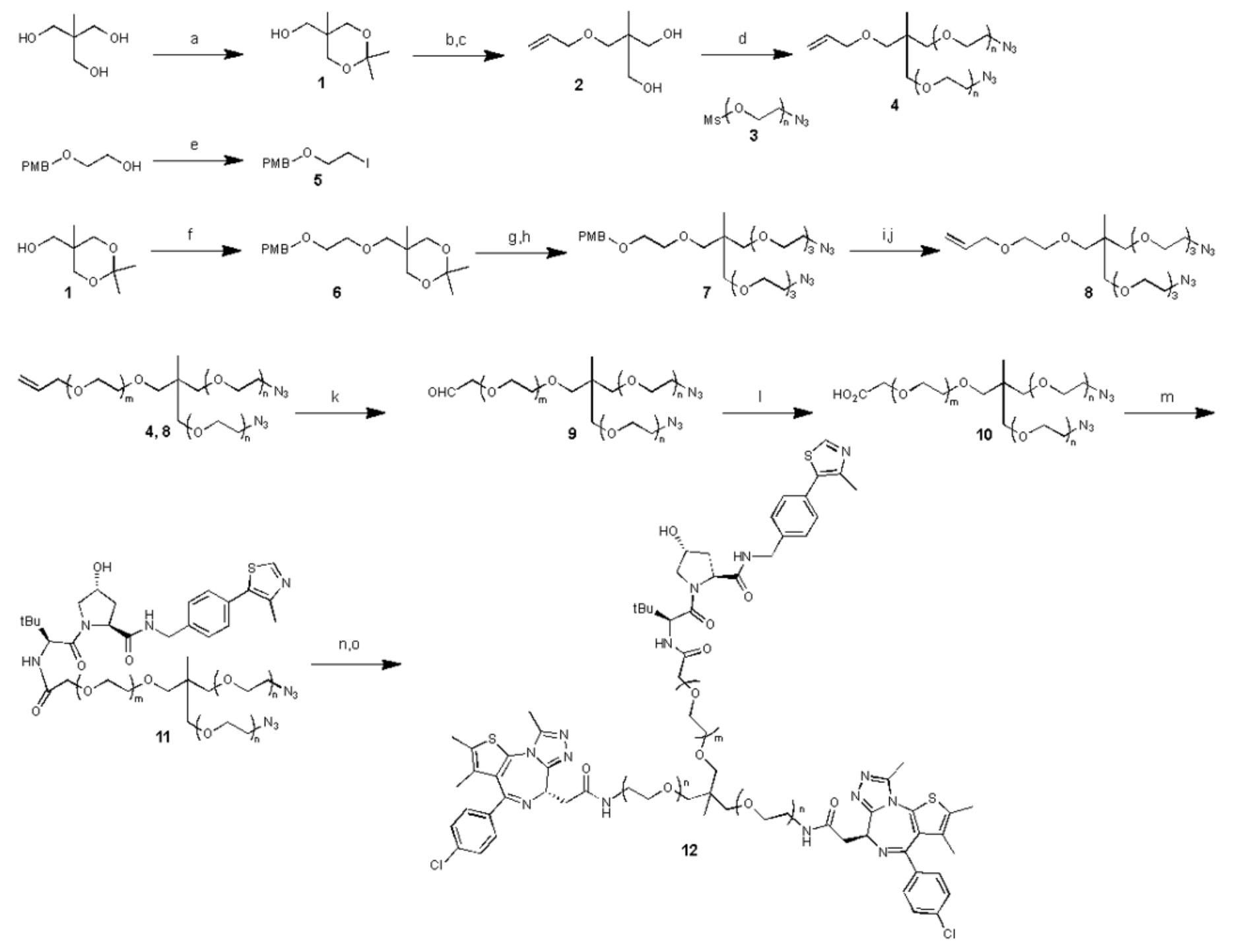

Figure 6

Scheme 1. Synthetic route to SIM1-SIM3. Reagents and conditions: (a) p-toluenesulfonic acid, acetone (96\%). (b-c) allyl bromide, KOH, TBAB, Toluene, H2O. TFA, methanol, H2O ( $86 \%$ over two steps). (d) 3, $\mathrm{KOH}, \mathrm{TBAB}, \mathrm{Kl}, 1,4$-dioxane ( $\mathrm{n}=3 \mathrm{12} \%, \mathrm{n}=4$ 9\%). (e) PPh3, I2, imidazole, $\mathrm{CH} 2 \mathrm{CH} 2$ (82\%). (f) 5, NaH, DMF (14\%). (g) TFA, methanol, $\mathrm{H} 2 \mathrm{O}$ (89\%). (h) 3 (n=3), KOH, TBAB, KI, 1,4-dioxane (55\%). (i) DDQ, $\mathrm{CH} 2 \mathrm{CH} 2$, $\mathrm{H} 2 \mathrm{O}$ (91\%). (j) allyl bromide, $\mathrm{KOH}, 1$,4-dioxane (72\%). (k) OsO4, NalO4, 2,6-luthidine, 1,4-dioxane, H2O $(m=0 n=3,64 \%, m=1 n=3,76 \%, m=0 n=4,65 \%)$. (I) NaClO2, NaH2PO4, 2-methyl-2-butene, t-Butanol, H2O. (m) VH032 amine $\mathrm{HCl}, \mathrm{COMU}$, DIPEA, DMF ( $m=0 \mathrm{n}=3,52 \%$ over two steps). VH032 amine HCl, HATU, HOAt, DIPEA, DMF ( $m=1 n=3,43 \%$ over two steps, $m=0 n=4,29 \%$ over two steps). ( $n$ ) $10 \%$ Pd-C, methanol. (o) (+)-JQ1 carboxylic acid, COMU, DIPEA, DMF ( $m=0 n=3,54 \%$ over two steps, $m=1 n=3,53 \%$ over two steps). (+)-JQ1 carboxylic acid, HATU, HOAt, DIPEA, DMF ( $m=0 n=4,43 \%$ over two steps). PMB, $p$ methoxybenzyl; TBAB, tetrabutylammonium bromide; TFA, trifluoroacetic acid; DMF, N,Ndimethylformamide; DDQ, 2,3-dichloro-5,6-dicyano-1,4-benzoquinone; COMU, 1-[(1-(cyano-2-ethoxy-2oxoethylideneaminooxy) dimethylaminomorpholino)] uronium hexafluorophosphate; DIPEA, N,N- 
diisopropylethylamine; HATU, 1-[bis(dimethylamino)methylene]-1 H-1,2,3-triazolo[4,5-b]pyridinium 3-oxide hexafluorophosphate; HOAt, 1-hydroxy-7-azabenzotriazole.
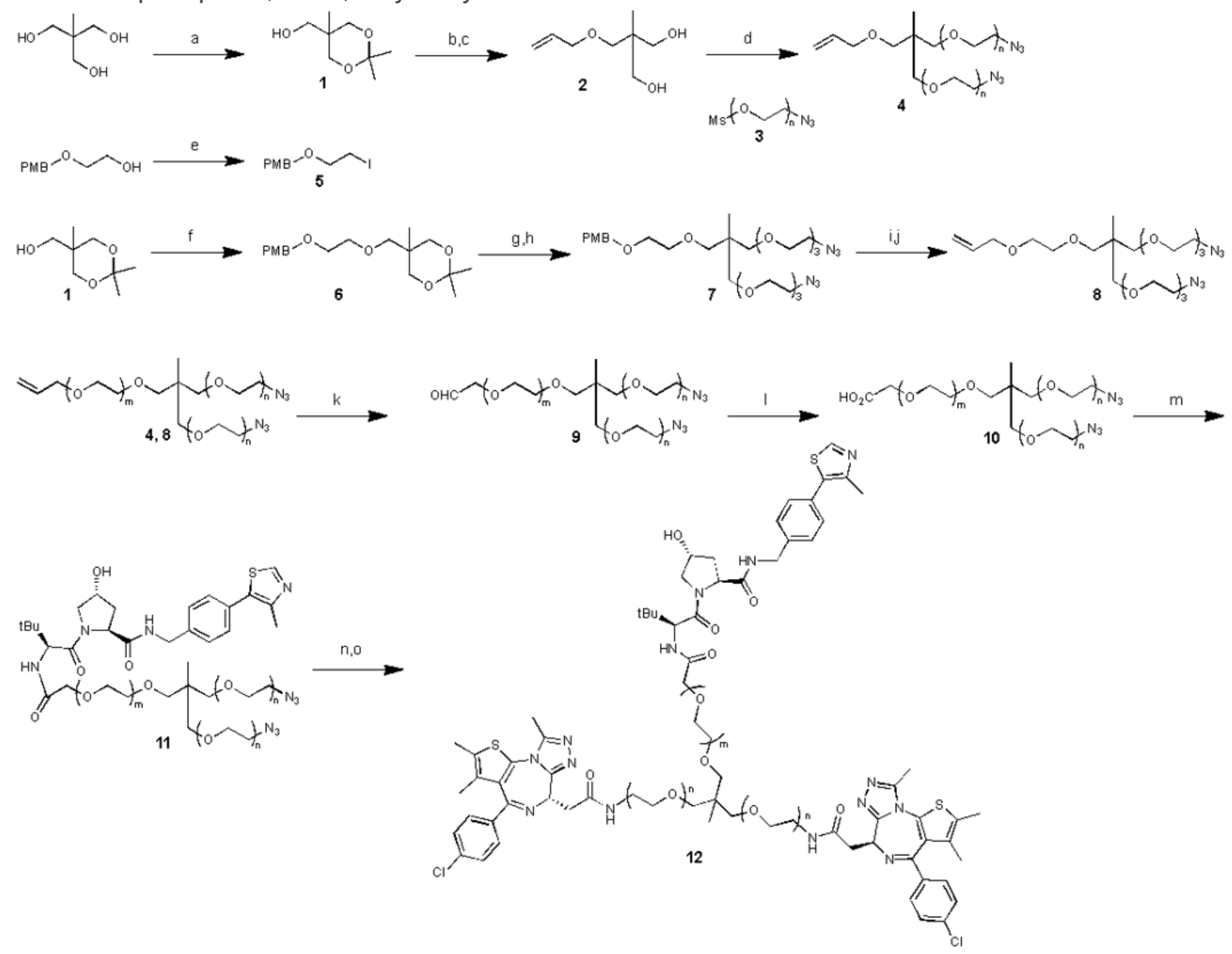

\section{Figure 6}

Scheme 1. Synthetic route to SIM1-SIM3. Reagents and conditions: (a) p-toluenesulfonic acid, acetone (96\%). (b-c) allyl bromide, KOH, TBAB, Toluene, H2O. TFA, methanol, H2O ( $86 \%$ over two steps). (d) 3, $\mathrm{KOH}, \mathrm{TBAB}, \mathrm{KI}, 1,4$-dioxane ( $\mathrm{n}=3 \mathrm{12} \%, \mathrm{n}=4$ 9\%). (e) PPh3, I2, imidazole, $\mathrm{CH} 2 \mathrm{CH} 2$ (82\%). (f) 5, NaH, DMF (14\%). (g) TFA, methanol, H2O (89\%). (h) 3 (n=3), KOH, TBAB, Kl, 1,4-dioxane (55\%). (i) DDQ, CH2CH2, $\mathrm{H} 2 \mathrm{O}$ (91\%). (j) allyl bromide, $\mathrm{KOH}, 1$,4-dioxane (72\%). (k) OsO4, NalO4, 2,6-luthidine, 1,4-dioxane, H2O ( $m=0 n=3,64 \%, m=1 n=3,76 \%, m=0 n=4,65 \%)$. (I) NaClO2, NaH2PO4, 2-methyl-2-butene, t-Butanol, H2O. (m) VH032 amine $\mathrm{HCl}, \mathrm{COMU}$, DIPEA, DMF ( $\mathrm{m}=0 \mathrm{n}=3,52 \%$ over two steps). VH032 amine $\mathrm{HCl}, \mathrm{HATU}$, HOAt, DIPEA, DMF ( $m=1 n=3,43 \%$ over two steps, $m=0 n=4,29 \%$ over two steps). ( $n$ ) $10 \%$ Pd-C, methanol. (o) (+)-JQ1 carboxylic acid, COMU, DIPEA, DMF ( $m=0 n=3,54 \%$ over two steps, $m=1 n=3,53 \%$ over two steps). (+)-JQ1 carboxylic acid, HATU, HOAt, DIPEA, DMF ( $m=0 \mathrm{n}=4,43 \%$ over two steps). PMB, $p$ methoxybenzyl; TBAB, tetrabutylammonium bromide; TFA, trifluoroacetic acid; DMF, N,Ndimethylformamide; DDQ, 2,3-dichloro-5,6-dicyano-1,4-benzoquinone; COMU, 1-[(1-(cyano-2-ethoxy-2- 
oxoethylideneaminooxy) dimethylaminomorpholino)] uronium hexafluorophosphate; DIPEA, N,Ndiisopropylethylamine; HATU, 1-[bis(dimethylamino)methylene]-1H-1,2,3-triazolo[4,5-b]pyridinium 3-oxide hexafluorophosphate; HOAt, 1-hydroxy-7-azabenzotriazole.

\section{Supplementary Files}

This is a list of supplementary files associated with this preprint. Click to download.

- SupplementaryNotechemistry.pdf

- SupplementaryNotechemistry.pdf

- ExtendedDataFiguresdocument.pdf

- ExtendedDataFiguresdocument.pdf 